\title{
Copyright
}

by

Bo Li

2007 
The Dissertation Committee for Bo Li

certifies that this is the approved version of the following dissertation:

\section{Applications of Noise Theory to Plasma Fluctuations}

Committee:

Richard D. Hazeltine, Supervisor

Roger D. Bengtson

Kenneth W. Gentle

Gary A. Hallock

James W. Van Dam 


\title{
Applications of Noise Theory to Plasma Fluctuations
}

\author{
by
}

Bo Li, B. S.

\section{Dissertation}

Presented to the Faculty of the Graduate School of

The University of Texas at Austin

in Partial Fulfillment

of the Requirements

for the Degree of

Doctor of Philosophy

The University of Texas at Austin

August 2007 
To the many people who have helped me along the road that has led to this dissertation.

In memory of my host families through the Christmas International House and the International Friendship programs. 


\section{Acknowledgments}

First of all, I would like to thank Richard Hazeltine, for being a great advisor, and for his support, guidance, and patience. I would also like to thank Roger Bengtson, Kenneth Gentle, Gary Hallock, and James Van Dam for serving on my dissertation committee, and for helpful discussions. I greatly appreciate helpful suggestions from Swadesh Mahajan, Mark Meier, Perry Phillips, and Edward Powers. I thank my fellow students, especially Kevin Lee, Dylan Miracle, Zhiping Yang, Guangye Chen, Jesse Pino and Tianchun Zhou for helpful conversations. This thesis has benefitted from the lectures on turbulent flow by David Bogard, fluid dynamics by Jack Swift, and cosmological fluctuations by Steven Weinberg.

I am deeply grateful to my parents and my relatives for their love and support. I am also grateful to Cheryl Banks, Daniel Harding, and their families for their precious friendship.

This work was supported by the U.S. Department of Energy.

Bo LI

The University of Texas at Austin

August 2007 


\title{
Applications of Noise Theory to Plasma Fluctuations
}

\author{
Publication No.
}

Bo Li, Ph.D.

The University of Texas at Austin, 2007

Supervisor: Richard D. Hazeltine

Fluctuation phenomena are important to many physical systems, such as the fusion plasma. Noise theory is used to study the time and space correlations of stationary Markovian fluctuations that are statistically homogeneous and isotropic. The relaxation of the fluctuations is modeled by the diffusion equation. The spatial correlations are modeled by the exponential decay. Based on these models, the correlation function and the power spectral density of random fluctuations are calculated. We find that the diffusion process can give rise to the decay of the correlation function and a broad frequency spectrum of random fluctuations. We also find that the fluctuation-induced transport coefficients may be estimated by the correlation length and the correlation time. The theoretical results are compared with the observed plasma density fluctuations from tokamak and helimak experiments. 


\section{Contents}

Acknowledgments $\quad$ v

Abstract vi vi vi vin

Chapter 1 Introduction to Noise Theory 1

1.1 Fluctuations ......................... 1

1.2 Stationary processes $\ldots \ldots \ldots \ldots 2$

1.3 The Wiener-Khintchin theorem .............. 4

1.4 Time-reversal symmetry . . . . . . . . . . . . . 8

1.5 Markovian relaxation . . . . . . . . . . . . . . 9

1.5.1 Markovian correlation function . . . . . . . . . . . . 11

1.5.2 Markovian power spectrum . . . . . . . . . . 12

$\begin{array}{lll}\text { Chapter } 2 & \text { Time and Space Correlations } & 13\end{array}$

2.1 Notation . . . . . . . . . . . . . . . . . . 13

2.2 Homogeneous statistics . . . . . . . . . . . . . . . . . . 14

2.2.1 The Wiener-Khintchin theorem _. . . . . . . . . 15

2.2.2 Wavenumber spectrum . . . . . . . . . . . . . 18

2.2.3 Wavenumber-frequency spectrum . . . . . . . . . 19

2.3 Isotropic statistics $\ldots \ldots \ldots \ldots \ldots$

2.4 Energy spectrum . . . . . . . . . . . . . . . . . 23 
2.5 Markovian noise spectrum _. . . . . . . . . . . . 24

$\begin{array}{lll}\text { Chapter } 3 & \text { Diffusion Model } & 28\end{array}$

3.1 Diffusion equation . . . . . . . . . . . . . . . 28

3.2 Delta function model . . . . . . . . . . . . . . . . . . . . . 30

3.3 Exponential decay model . . . . . . . . . . . . . . . . 33

3.4 Energy spectrum of exponential decay . . . . . . . . . . . . . . . 34

3.5 Autopower spectrum . . . . . . . . . . . . . . . . . . 37

3.6 Autocorrelation function . . . . . . . . . . . . . . . 42

3.7 Oscillating decay . . . . . . . . . . . . . . . . . 46

$\begin{array}{lll}\text { Chapter } 4 & \text { Convection-Diffusion Model } & 49\end{array}$

4.1 Convection-diffusion equation . . . . . . . . . . . . . . . . 49

4.2 Anisotropy in magnetized plasmas . . . . . . . . . . . . . 50

Chapter 5 Comparison with Experiment 52

5.1 Fluctuations in magnetized plasmas . . . . . . . . . . . 52

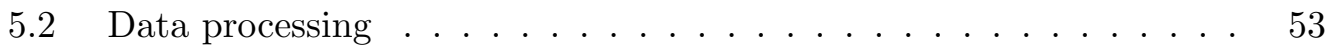

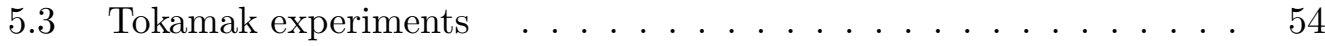

5.4 Helimak experiments . . . . . . . . . . . . . . . . 55

5.5 Discussion ............................. 61

$\begin{array}{lll}\text { Chapter } 6 & \text { Summary and Conclusions } & 62\end{array}$

$\begin{array}{lr}\text { Bibliography } & 66\end{array}$

$\begin{array}{ll}\text { Vita } & 67\end{array}$ 


\section{Chapter 1}

\section{Introduction to Noise Theory}

The fluctuation-induced transport of particles and energy is important to plasma confinement in a fusion device $[1,2,3]$. Various methods have been developed for solving fluctuation problems $[4,5,6,7,8,9,10,11]$. In this work, we treat random fluctuations as noise and use noise theory to study the statistical properties of stationary Markovian random fluctuations.

In this chapter, we review the methods of solving Markovian noise problems $[12,13]$. In order to quantify the temporal correlations of stationary processes, we define two important statistical quantities: the correlation function and the power spectral density. After introducing the Wiener-Khintchin theorem, we describe the relaxation of Markov processes in terms of the conditional mean. Finally, we calculate the correlation function and the power spectrum of Markovian noise.

\section{$1.1 \quad$ Fluctuations}

We consider a system described by a set of macroscopic quantities $\boldsymbol{a}(t)=\left\{a_{n}(t)\right\}$, each of which is treated as a stochastic process. The equilibrium or steady-state value of the system is given by the mean value $\langle\boldsymbol{a}(t)\rangle$, where the angle brackets 
$\langle\cdots\rangle$ denote an ensemble average. The deviations from the mean value are called the fluctuations

$$
\boldsymbol{\alpha}(t)=\boldsymbol{a}(t)-\langle\boldsymbol{a}(t)\rangle
$$

By definition, the fluctuations have zero average value

$$
\langle\boldsymbol{\alpha}(t)\rangle=0 .
$$

The variance is defined by the mean square value of the fluctuations: $\boldsymbol{\sigma}^{2}=\left\langle\boldsymbol{\alpha}^{2}\right\rangle$, where $\boldsymbol{\sigma}$ is the standard deviation, i.e., the root mean square deviations. The ratio of the standard deviation $\boldsymbol{\sigma}$ to the mean value $\langle\boldsymbol{a}\rangle$ gives the fluctuation level. When $\boldsymbol{\alpha}$ represents the fractional fluctuations, that is, $\boldsymbol{\alpha}=(\boldsymbol{a}-\langle\boldsymbol{a}\rangle) /\langle\boldsymbol{a}\rangle$, the fluctuation level is equal to the standard deviation $\boldsymbol{\sigma}$.

\section{$1.2 \quad$ Stationary processes}

A stochastic process $\alpha_{n}(t)$ is a family of random variables, ordered and labeled by time. A distinctive feature of a family of random variables is that variables labeled by different times may be correlated. The correlations between two times can be quantified by the correlation function

$$
\left\langle\boldsymbol{\alpha}(t) \boldsymbol{\alpha}\left(t^{\prime}\right)\right\rangle=\int d \boldsymbol{\alpha} d \boldsymbol{\alpha}^{\prime} P\left(\boldsymbol{\alpha}, t, \boldsymbol{\alpha}^{\prime}, t^{\prime}\right) \boldsymbol{\alpha} \boldsymbol{\alpha}^{\prime}
$$

where the joint probability $P\left(\boldsymbol{\alpha}, t, \boldsymbol{\alpha}^{\prime}, t^{\prime}\right)$ measures the probability of observing the event $\boldsymbol{\alpha}$ at time $t$ and the event $\boldsymbol{\alpha}^{\prime}$ at time $t^{\prime}$. It is very convenient to express the joint probability as

$$
P\left(\boldsymbol{\alpha}, t, \boldsymbol{\alpha}^{\prime}, t^{\prime}\right)=P\left(\boldsymbol{\alpha}, t \mid \boldsymbol{\alpha}^{\prime}, t^{\prime}\right) P\left(\boldsymbol{\alpha}^{\prime}, t^{\prime}\right),
$$


where the probability density function $P(\boldsymbol{\alpha}, t)$ measures the probability of observing the event $\boldsymbol{\alpha}$ at time $t$, and the conditional probability $P\left(\boldsymbol{\alpha}, t \mid \boldsymbol{\alpha}^{\prime}, t^{\prime}\right)$ measures the probability of observing the state $\boldsymbol{\alpha}$ at time $t$, given that one has observed the state $\boldsymbol{\alpha}^{\prime}$ at time $t^{\prime}$. Inserting Eq. (1.4) into Eq. (1.3), we have

$$
\left\langle\boldsymbol{\alpha}(t) \boldsymbol{\alpha}\left(t^{\prime}\right)\right\rangle=\int d \boldsymbol{\alpha} d \boldsymbol{\alpha}^{\prime} P\left(\boldsymbol{\alpha}, t \mid \boldsymbol{\alpha}^{\prime}, t^{\prime}\right) P\left(\boldsymbol{\alpha}^{\prime}, t^{\prime}\right) \boldsymbol{\alpha} \boldsymbol{\alpha}^{\prime}
$$

For stationary processes, probability distributions are invariant under translations in time, so that the probability density function $P(\boldsymbol{\alpha}, t)$ is independent of time $t$ and the conditional probability $P\left(\boldsymbol{\alpha}, t \mid \boldsymbol{\alpha}^{\prime}, t^{\prime}\right)$ depends only on time differences $\tau=t-t^{\prime}$. Hence we can write Eq. (1.5) as

$$
\boldsymbol{C}(\tau)=\left\langle\boldsymbol{\alpha}(t) \boldsymbol{\alpha}\left(t^{\prime}\right)\right\rangle=\int d \boldsymbol{\alpha} d \boldsymbol{\alpha}^{\prime} P\left(\boldsymbol{\alpha}, \tau \mid \boldsymbol{\alpha}^{\prime}\right) P\left(\boldsymbol{\alpha}^{\prime}\right) \boldsymbol{\alpha} \boldsymbol{\alpha}^{\prime}
$$

where the correlation matrix $\boldsymbol{C}(\tau)$ has components

$$
C_{n m}(\tau)=\left\langle\alpha_{n}(t) \alpha_{m}\left(t^{\prime}\right)\right\rangle=\left\langle\alpha_{n}(t) \alpha_{m}(t-\tau)\right\rangle
$$

Exchanging the indices and replacing $\tau$ with $-\tau$ in Eq. (1.7), we have

$$
C_{m n}(-\tau)=\left\langle\alpha_{m}(t) \alpha_{n}(t+\tau)\right\rangle
$$

For stationary statistics, we see that

$$
C_{n m}(\tau)=C_{m n}(-\tau) \text { or } \boldsymbol{C}(\tau)=\boldsymbol{C}^{T}(-\tau)
$$

where the superscript $T$ indicates the transpose matrix. It follows from Eq. (1.9) that the single-time correlation matrix $\boldsymbol{C}(\tau=0)$ is symmetric, and the autocorrelation function $C_{n n}(\tau)$ is even in $\tau$. 
Finally, we assume that the stationary process is ergodic, so that the ensemble average is equal to the time average. For instance,

$$
\left\langle\alpha_{n}(t) \alpha_{m}(t-\tau)\right\rangle=\lim _{T \rightarrow \infty} \frac{1}{T} \int_{0}^{T} d t \alpha_{n}(t) \alpha_{m}(t-\tau)
$$

where $T$ is the record length of the time series data.

\subsection{The Wiener-Khintchin theorem}

For stationary processes, the real quantity $\alpha_{n}(t)$ can be expressed as Fourier integrals

$$
\alpha_{n}(t)=\int_{-\infty}^{\infty} \frac{d \omega}{2 \pi} e^{i \omega t} \alpha_{n}(\omega)
$$

where $\omega=2 \pi f$ is a real frequency, and

$$
\alpha_{n}(\omega)=\int_{-\infty}^{\infty} d t e^{-i \omega t} \alpha_{n}(t)
$$

denotes the Fourier transform of $\alpha_{n}(t)$. It follows from Eq. (1.12) that

$$
\alpha_{n}^{*}(\omega)=\alpha_{n}(-\omega)
$$

where the asterisk denotes the complex conjugate, and

$$
\left\langle\alpha_{n}(\omega)\right\rangle=\int_{-\infty}^{\infty} d t e^{-i \omega t}\left\langle\alpha_{n}(t)\right\rangle
$$

Since the fluctuations $\alpha_{n}(t)$ have zero mean $\left\langle\alpha_{n}(t)\right\rangle=0$, the random quantity $\alpha_{n}(\omega)$ also have zero mean $\left\langle\alpha_{n}(\omega)\right\rangle=0$.

Let us consider the average of the product of two real quantities $\alpha_{n}(t)$ and $\alpha_{m}\left(t^{\prime}\right)$. It turns out to be very convenient to use Eq. (1.11) for $\alpha_{n}(t)$ and its complex 
conjugate for $\alpha_{m}\left(t^{\prime}\right)$, so that

$$
\left\langle\alpha_{n}(t) \alpha_{m}\left(t^{\prime}\right)\right\rangle=\int_{-\infty}^{\infty} \frac{d \omega}{2 \pi} \int_{-\infty}^{\infty} \frac{d \omega^{\prime}}{2 \pi} e^{i\left(\omega t-\omega^{\prime} t^{\prime}\right)}\left\langle\alpha_{n}(\omega) \alpha_{m}^{*}\left(\omega^{\prime}\right)\right\rangle
$$

For stationary processes, $\left\langle\alpha_{n}(t) \alpha_{m}\left(t^{\prime}\right)\right\rangle$ should be a function only of $\tau=t-t^{\prime}$. This immediately tells us that $\left\langle\alpha_{n}(\omega) \alpha_{m}^{*}\left(\omega^{\prime}\right)\right\rangle$ must be proportional to a delta function, $\delta\left(\omega-\omega^{\prime}\right)$. That is, we can write

$$
\left\langle\alpha_{n}(\omega) \alpha_{m}^{*}\left(\omega^{\prime}\right)\right\rangle=S_{n m}(\omega) 2 \pi \delta\left(\omega-\omega^{\prime}\right)
$$

where $S_{n m}(\omega)$ is the power spectral density, also called the power spectrum. Since $\omega=2 \pi f$, Eq. (1.16) can be rewritten as

$$
\left\langle\alpha_{n}(f) \alpha_{m}^{*}\left(f^{\prime}\right)\right\rangle=S_{n m}(f) \delta\left(f-f^{\prime}\right) .
$$

In practice, the power spectral density is defined by

$$
S_{n m}(\omega)=\lim _{T \rightarrow \infty} \frac{1}{T}\left\langle\alpha_{n}(\omega, T) \alpha_{m}^{*}(\omega, T)\right\rangle
$$

where $T$ is the record length of the time series data, and

$$
\alpha_{n}(\omega, T)=\int_{-T / 2}^{T / 2} d t e^{-i \omega t} \alpha_{n}(t)
$$

Inserting Eq. (1.16) into Eq. (1.15), we have

$$
\left\langle\alpha_{n}(t) \alpha_{m}\left(t^{\prime}\right)\right\rangle=C_{n m}(\tau)=\int_{-\infty}^{\infty} \frac{d \omega}{2 \pi} e^{i \omega \tau} S_{n m}(\omega)
$$

Eq. (1.20) tells us that the power spectrum is equal to the Fourier transform of the 
correlation function. That is, we can write

$$
S_{n m}(\omega)=\int_{-\infty}^{\infty} d \tau e^{-i \omega \tau} C_{n m}(\tau)=\int_{-\infty}^{\infty} d \tau[\cos (\omega \tau)-i \sin (\omega \tau)] C_{n m}(\tau)
$$

Eqs. (1.20) or (1.21) are known as the Wiener-Khintchin theorem.

It follows from Eq. (1.21) that

$$
\boldsymbol{S}^{T *}(\omega)=\boldsymbol{S}^{T}(-\omega)=\int_{-\infty}^{\infty} d \tau e^{i \omega \tau} \boldsymbol{C}^{T}(\tau)=\int_{-\infty}^{\infty} d \tau^{\prime} e^{-i \omega \tau^{\prime}} \boldsymbol{C}^{T}\left(-\tau^{\prime}\right)
$$

where $\tau^{\prime}=-\tau$. Since $\boldsymbol{C}(\tau)=\boldsymbol{C}^{T}(-\tau)$, we see that $\boldsymbol{S}(\omega)$ is a Hermitian matrix

$$
\boldsymbol{S}(\omega)=\boldsymbol{S}^{T *}(\omega)=\boldsymbol{S}^{T}(-\omega) \text { or } S_{n m}(\omega)=S_{m n}^{*}(\omega)=S_{m n}(-\omega) .
$$

Eq. (1.21) shows that the real part of $S_{n m}(\omega)$ is even in $\omega$ and the imaginary part of $S_{n m}(\omega)$ is odd in $\omega$. When the correlation function $C_{n m}(\tau)$ is even in $\tau$, the power spectrum $S_{n m}(\omega)$ is a real, even function of frequency. When the correlation function $C_{n m}(\tau)$ is neither even nor odd in $\tau$, the power spectrum $S_{n m}(\omega)$ is complex:

$$
S_{n m}(\omega)=\left|S_{n m}(\omega)\right| e^{i \theta_{n m}(\omega)},
$$

where $\theta_{n m}(\omega)$ is called the phase spectrum, and the magnitude $\left|S_{n m}(\omega)\right|$ defines the coherence spectrum

$$
\gamma_{n m}^{2}(\omega)=\frac{\left|S_{n m}(\omega)\right|^{2}}{S_{m m}(\omega) S_{n n}(\omega)}
$$

where the coherence $0 \leq \gamma_{n m}^{2}(\omega) \leq 1$.

It follows from Eq. (1.20) that the single-time correlation matrix

$$
\left\langle\alpha_{n}(t) \alpha_{m}(t)\right\rangle=C_{n m}(\tau=0)=\int_{-\infty}^{\infty} \frac{d \omega}{2 \pi} S_{n m}(\omega)
$$

One can take $\alpha_{n}(t)$ to measure the voltage, so that $\left\langle\alpha_{n}^{2}(t)\right\rangle$ is the average power 
dissipated in a resistor of one Ohm. It follows from Eq. (1.26) that the area under the autopower spectral density is equal to the average power of the fluctuations:

$$
\left\langle\alpha_{n}^{2}(t)\right\rangle=C_{n n}(\tau=0)=\int_{-\infty}^{\infty} \frac{d \omega}{2 \pi} S_{n n}(\omega)
$$

It is clear that the autopower spectral density $S_{n n}(\omega)$ indicates the frequency distribution of the fluctuation energy and $S_{n n}(\omega) d \omega$ specifies the fluctuation power in the frequency interval $d \omega$. Setting the index $m=n$ in Eq. (1.16), we see that the autopower spectrum $S_{n n}(\omega)$ is real and positive. Setting the index $m=n$ in Eq. (1.23), we see that the autopower spectrum $S_{n n}(\omega)$ is even in $\omega$.

Finally, we write Eq. (1.21) as

$$
\boldsymbol{S}(\omega)=\boldsymbol{S}_{+}(\omega)+\boldsymbol{S}_{-}(\omega)
$$

defining

$$
\boldsymbol{S}_{+}(\omega)=\int_{0}^{\infty} d \tau e^{-i \omega \tau} \boldsymbol{C}(\tau)
$$

and

$$
\boldsymbol{S}_{-}(\omega)=\int_{-\infty}^{0} d \tau e^{-i \omega \tau} \boldsymbol{C}(\tau)
$$

It follows from Eq. (1.29) that

$$
\boldsymbol{S}_{+}^{T *}(\omega)=\boldsymbol{S}_{+}^{T}(-\omega)=\int_{0}^{\infty} d \tau e^{i \omega \tau} \boldsymbol{C}^{T}(\tau)=\int_{-\infty}^{0} d \tau^{\prime} e^{-i \omega \tau^{\prime}} \boldsymbol{C}^{T}\left(-\tau^{\prime}\right)
$$

where $\tau^{\prime}=-\tau$. Since $\boldsymbol{C}(\tau)=\boldsymbol{C}^{T}(-\tau)$, we see that

$$
\boldsymbol{S}_{-}(\omega)=\boldsymbol{S}_{+}^{T *}(\omega)=\boldsymbol{S}_{+}^{T}(-\omega)
$$


Thus Eq. (1.28) can be rewritten as

$$
\boldsymbol{S}(\omega)=\boldsymbol{S}_{+}(\omega)+\boldsymbol{S}_{+}^{* T}(\omega)
$$

Note that this expression for the power spectrum satisfies the condition Eq. (1.23).

\section{$1.4 \quad$ Time-reversal symmetry}

Following Onsager [12], time-reversal symmetry implies that the event $\alpha_{n}$, followed $\tau$ seconds later by the event $\alpha_{m}$, will occur just as often as the event $\alpha_{m}$, followed $\tau$ seconds later by the event $\alpha_{n}$ :

$$
\left\langle\alpha_{n}(t) \alpha_{m}(t-\tau)\right\rangle=\left\langle\alpha_{n}(t) \alpha_{m}(t+\tau)\right\rangle
$$

That is, in the case of time-reversal symmetry the correlation function is even in $\tau$ :

$$
C_{n m}(\tau)=C_{n m}(-\tau) \text { or } \boldsymbol{C}(\tau)=\boldsymbol{C}(-\tau)
$$

Since $\boldsymbol{C}(-\tau)=\boldsymbol{C}^{T}(\tau)$, we see that the matrix $\boldsymbol{C}(\tau)$ becomes symmetric:

$$
\boldsymbol{C}(\tau)=\boldsymbol{C}^{T}(\tau)
$$

and the matrix $\boldsymbol{S}_{+}(\omega)$ also becomes symmetric:

$$
\boldsymbol{S}_{+}^{T}(\omega)=\int_{0}^{\infty} d \tau e^{-i \omega \tau} \boldsymbol{C}^{T}(\tau)=\boldsymbol{S}_{+}(\omega)
$$

Thus Eq. (1.33) becomes

$$
\boldsymbol{S}(\omega)=\boldsymbol{S}_{+}(\omega)+\boldsymbol{S}_{+}^{*}(\omega)=2 \operatorname{Re} \boldsymbol{S}_{+}(\omega)
$$


where Re indicates taking the real part. It is important to note that in the case of time-reversal symmetry the power spectrum is always real.

\subsection{Markovian relaxation}

We can consider $\boldsymbol{\alpha}=0$ to be the equilibrium or steady-state value, to which $\boldsymbol{\alpha}(t)$ will relax after the initial perturbation $\boldsymbol{\alpha}(t=0)=\boldsymbol{\alpha}_{0}$. It is natural to describe this relaxation process by means of the conditional mean. The conditional mean is defined by

$$
\langle\boldsymbol{\alpha}(t)\rangle_{0}=\int d \boldsymbol{\alpha} P\left(\boldsymbol{\alpha}, t \mid \boldsymbol{\alpha}_{0}\right) \boldsymbol{\alpha}
$$

where $\langle\cdots\rangle_{0}$ denotes the conditional mean. Recall that the conditional probability $P\left(\boldsymbol{\alpha}, t \mid \boldsymbol{\alpha}_{0}\right)$ measures the probability of observing the value $\boldsymbol{\alpha}$ at time $t>0$, given the initial perturbation $\boldsymbol{\alpha}_{0}$. At time $t=0$, we expect $P\left(\boldsymbol{\alpha}, t \mid \boldsymbol{\alpha}_{0}\right) \rightarrow \delta\left(\boldsymbol{\alpha}-\boldsymbol{\alpha}_{0}\right)$, so that the conditional mean becomes the initial perturbation

$$
\langle\boldsymbol{\alpha}(0)\rangle_{0}=\boldsymbol{\alpha}_{0}
$$

For $t \rightarrow \infty$ we expect $P\left(\boldsymbol{\alpha}, t \mid \boldsymbol{\alpha}_{0}\right) \rightarrow P(\boldsymbol{\alpha}, t)$, so that the conditional mean becomes an ensemble average of the fluctuations

$$
\langle\boldsymbol{\alpha}(t)\rangle_{0} \rightarrow\langle\boldsymbol{\alpha}(t)\rangle=0
$$

The conditional mean Eq. (1.39) can be rewritten as

$$
\langle\boldsymbol{\alpha}(t+\Delta t)\rangle_{0}=\int d \boldsymbol{\alpha} P\left(\boldsymbol{\alpha}, t+\Delta t \mid \boldsymbol{\alpha}_{0}\right) \boldsymbol{\alpha}
$$

For a stationary Markov process, the conditional probability must obey the 
Chapman-Kolmogorov equation

$$
P\left(\boldsymbol{\alpha}, t+\Delta t \mid \boldsymbol{\alpha}_{0}\right)=\int d \boldsymbol{\alpha}^{\prime} P\left(\boldsymbol{\alpha}^{\prime}, t \mid \boldsymbol{\alpha}_{0}\right) P\left(\boldsymbol{\alpha}, \Delta t \mid \boldsymbol{\alpha}^{\prime}\right)
$$

where $P\left(\boldsymbol{\alpha}, \Delta t \mid \boldsymbol{\alpha}^{\prime}\right)$ measures the probability of observing the transition from the state $\boldsymbol{\alpha}^{\prime}$ to the state $\boldsymbol{\alpha}$ in the time interval $\Delta t$. Inserting Eq. (1.43) into Eq. (1.42), we have

$$
\langle\boldsymbol{\alpha}(t+\Delta t)\rangle_{0}=\int d \boldsymbol{\alpha}^{\prime} P\left(\boldsymbol{\alpha}^{\prime}, t \mid \boldsymbol{\alpha}_{0}\right) \int d \boldsymbol{\alpha} P\left(\boldsymbol{\alpha}, \Delta t \mid \boldsymbol{\alpha}^{\prime}\right) \boldsymbol{\alpha}
$$

When the time interval $\Delta t$ and the initial perturbation $\boldsymbol{\alpha}_{0}$ are sufficiently small, the response of the system to the perturbations is assumed to take the form

$$
\int d \boldsymbol{\alpha} P\left(\boldsymbol{\alpha}, \Delta t \mid \boldsymbol{\alpha}^{\prime}\right) \boldsymbol{\alpha}=\boldsymbol{\alpha}^{\prime}-\Delta t \boldsymbol{\Lambda} \cdot \boldsymbol{\alpha}^{\prime}
$$

where $\boldsymbol{\Lambda}$ is called the response matrix. Note that $\boldsymbol{\Lambda}$ is independent of time $t$. Inserting Eq. (1.45) into Eq. (1.44), we obtain

$$
\langle\boldsymbol{\alpha}(t+\Delta t)\rangle_{0}=\langle\boldsymbol{\alpha}(t)\rangle_{0}-\Delta t \boldsymbol{\Lambda} \cdot\langle\boldsymbol{\alpha}(t)\rangle_{0}
$$

For $\Delta t \rightarrow 0$, Eq. (1.46) becomes

$$
\frac{\partial}{\partial t}\langle\boldsymbol{\alpha}(t)\rangle_{0}=-\boldsymbol{\Lambda} \cdot\langle\boldsymbol{\alpha}(t)\rangle_{0}
$$

or

$$
\frac{\partial}{\partial t}\left\langle\alpha_{n}(t)\right\rangle_{0}=-\Lambda_{n m}\left\langle\alpha_{m}(t)\right\rangle_{0}
$$

Eq. (1.47) describes the relaxation of the conditional mean. The formal solution of Eq. (1.47) is

$$
\langle\boldsymbol{\alpha}(t)\rangle_{0}=e^{-\boldsymbol{\Lambda} t} \cdot \boldsymbol{\alpha}_{0}, \quad \text { for } t>0
$$

Note that relaxation implies that every element of the matrix $\boldsymbol{\Lambda}$ should be positive, 
so that the solution Eq. (1.48) satisfies the conditions Eqs. (1.40) and (1.41).

\subsubsection{Markovian correlation function}

If we let $t^{\prime}=0, \boldsymbol{\alpha}^{\prime}=\boldsymbol{\alpha}_{0}$, and $\tau=t$ in Eq. (1.6), we have

$$
\langle\boldsymbol{\alpha}(t) \boldsymbol{\alpha}(0)\rangle=\int d \boldsymbol{\alpha} d \boldsymbol{\alpha}_{0} P\left(\boldsymbol{\alpha}, t \mid \boldsymbol{\alpha}_{0}\right) P\left(\boldsymbol{\alpha}_{0}\right) \boldsymbol{\alpha} \boldsymbol{\alpha}_{0}
$$

Using Eq. (1.39) we can express the correlation function Eq. (1.49) in terms of the conditional mean:

$$
\langle\boldsymbol{\alpha}(t) \boldsymbol{\alpha}(0)\rangle=\int d \boldsymbol{\alpha}_{0}\langle\boldsymbol{\alpha}(t)\rangle_{0} P\left(\boldsymbol{\alpha}_{0}\right) \boldsymbol{\alpha}_{0}
$$

Inserting Eq. (1.48) into Eq. (1.50), we obtain the formal expression

$$
\langle\boldsymbol{\alpha}(t) \boldsymbol{\alpha}(0)\rangle=e^{-\boldsymbol{\Lambda} t} \cdot\langle\boldsymbol{\alpha}(0) \boldsymbol{\alpha}(0)\rangle, \quad \text { for } t>0
$$

For stationary processes, the single-time correlation function is independent of time

$$
\boldsymbol{C}(\tau=0)=\langle\boldsymbol{\alpha}(t) \boldsymbol{\alpha}(t)\rangle=\langle\boldsymbol{\alpha}(0) \boldsymbol{\alpha}(0)\rangle
$$

From Eq. (1.7) we see that $\langle\boldsymbol{\alpha}(t) \boldsymbol{\alpha}(0)\rangle$ is the correlation function for the case $t^{\prime}=0$ and $\tau=t$. Hence we can write Eq. (1.51) as

$$
\boldsymbol{C}(\tau)=e^{-\boldsymbol{\Lambda} \tau} \cdot \boldsymbol{C}(\tau=0), \quad \text { for } \tau>0
$$

In the case of time-reversal symmetry, the correlation function is even in $\tau$. Thus Eq. (1.53) becomes

$$
\boldsymbol{C}(\tau)=e^{-\boldsymbol{\Lambda}|\tau|} \cdot \boldsymbol{C}(\tau=0)
$$

Note that the slope of the even function $\boldsymbol{C}(\tau)$ should be zero at $\tau=0$, but the slope 
of Markovian correlation function Eq. (1.54) does not vanish at $\tau=0$. The slope discontinuity at the origin is a consequence of the Markovian approximation [12].

\subsubsection{Markovian power spectrum}

Inserting Eq. (1.53) into Eq. (1.29), we obtain the formal expression

$$
\boldsymbol{S}_{+}(\omega)=(\boldsymbol{\Lambda}+i \omega \boldsymbol{I})^{-1} \cdot \boldsymbol{C}(\tau=0)
$$

or

$$
(\boldsymbol{\Lambda}+i \omega \boldsymbol{I}) \cdot \boldsymbol{S}_{+}(\omega)=\boldsymbol{C}(\tau=0)
$$

where $\boldsymbol{I}$ is the unit matrix. The response matrix $\boldsymbol{\Lambda}$ is real and the matrix $\boldsymbol{C}(\tau=0)$ is symmetric, so by taking the transpose and complex conjugate of Eq. (1.56), we obtain the formal expression

$$
\boldsymbol{S}_{-}(\omega)=\boldsymbol{S}_{+}^{T *}(\omega)=\boldsymbol{C}(\tau=0) \cdot\left(\boldsymbol{\Lambda}^{T}-i \omega \boldsymbol{I}\right)^{-1}
$$

or

$$
\boldsymbol{S}_{-}(\omega) \cdot\left(\boldsymbol{\Lambda}^{T}-i \omega \boldsymbol{I}\right)=\boldsymbol{C}(\tau=0) .
$$

Inserting Eqs. (1.55) and (1.57) into Eq. (1.28), we have

$$
\boldsymbol{S}(\omega)=(\boldsymbol{\Lambda}+i \omega \boldsymbol{I})^{-1} \cdot \boldsymbol{C}(\tau=0)+\boldsymbol{C}(\tau=0) \cdot\left(\boldsymbol{\Lambda}^{T}-i \omega \boldsymbol{I}\right)^{-1} .
$$

Thus the power spectrum is known in terms of the response matrix $\boldsymbol{\Lambda}$ and the single-time correlation function $\boldsymbol{C}(\tau=0)$.

In the case of time-reversal symmetry, it follows from Eqs. (1.38) and (1.55) that the power spectrum

$$
\boldsymbol{S}(\omega)=2 \operatorname{Re} \boldsymbol{S}_{+}(\omega)=2 \operatorname{Re}(\boldsymbol{\Lambda}+i \omega \boldsymbol{I})^{-1} \cdot \boldsymbol{C}(\tau=0)
$$




\section{Chapter 2}

\section{Time and Space Correlations}

In the turbulent state, the system is irregular in both space and time. Therefore, we need to study both the spatial and temporal correlations of random fluctuations. In this chapter, we will introduce some average quantities for the statistical description of homogeneous isotropic fluctuations. We will also derive Markovian noise spectra of homogeneous fluctuations.

\section{$2.1 \quad$ Notation}

Up to now we have treated the variables $\boldsymbol{\alpha}=\left\{\alpha_{n}\right\}$ as a vector with a finite number of discrete components. In this work, we consider the fluctuations in a single quantity, such as particle number density, at different spatial points. Hence in the case of spatial variation, we simply replace the discrete index $n$ by the continuous index $\boldsymbol{x}$. For instance,

$$
\boldsymbol{\alpha}(t) \rightarrow \alpha_{n}(t) \rightarrow \alpha(\boldsymbol{x}, t)
$$

Another change in the formalism is that sums over the discrete index are replaced by integrals over the spatial coordinate. For example, the relaxation of the conditional 
mean Eq. (1.47) becomes

$$
\frac{\partial}{\partial t}\langle\alpha(\boldsymbol{x}, t)\rangle_{0}+\int d^{3} x^{\prime} \Lambda\left(\boldsymbol{x}, \boldsymbol{x}^{\prime}\right)\left\langle\alpha\left(\boldsymbol{x}^{\prime}, t\right)\right\rangle_{0}=0
$$

\subsection{Homogeneous statistics}

For homogeneous statistics, probability distributions are invariant under translations in space, so that the statistical properties of homogeneous fluctuations are independent of position. For instance, the spatial correlations between two points can only depend on the spatial coordinate differences $\boldsymbol{r}=\boldsymbol{x}-\boldsymbol{x}^{\prime}$.

We will assume that the fluctuations are homogeneous in space. The assumption of spatial homogeneity approximates the true statistics if the length scales associated with the fluctuations are short compared to equilibrium length scales of the system. We will also assume that the fluctuations are stationary in time, so that the temporal correlations between two times can only depend on the time coordinate differences $\tau=t-t^{\prime}$.

Hence for stationary, homogeneous fluctuations we can write the correlation function as

$$
C(\boldsymbol{r}, \tau)=\left\langle\alpha(\boldsymbol{x}, t) \alpha\left(\boldsymbol{x}^{\prime}, t^{\prime}\right)\right\rangle=\langle\alpha(\boldsymbol{x}, t) \alpha(\boldsymbol{x}-\boldsymbol{r}, t-\tau)\rangle .
$$

In particular, the mean square deviations are independent of time $t$ and position $\boldsymbol{x}$. That is,

$$
C(\boldsymbol{r}=0, \tau=0)=\left\langle\alpha^{2}(\boldsymbol{x}, t)\right\rangle=\left\langle\alpha^{2}\right\rangle=\sigma^{2},
$$

where the standard deviation $\sigma$ is a constant. 


\subsubsection{The Wiener-Khintchin theorem}

For stationary processes, the fluctuations $\alpha(\boldsymbol{x}, t)$ may be written as temporal Fourier integrals

$$
\alpha(\boldsymbol{x}, t)=\int_{-\infty}^{\infty} \frac{d \omega}{2 \pi} e^{i \omega t} \alpha(\boldsymbol{x}, \omega)
$$

where $\omega=2 \pi f$ is a real frequency and

$$
\alpha(\boldsymbol{x}, \omega)=\int_{-\infty}^{\infty} d t e^{-i \omega t} \alpha(\boldsymbol{x}, t)
$$

denotes the temporal Fourier transform of $\alpha(\boldsymbol{x}, t)$.

The average of the product of two real quantities $\alpha(\boldsymbol{x}, t)$ and $\alpha\left(\boldsymbol{x}^{\prime}, t^{\prime}\right)$ can be written as

$$
\left\langle\alpha(\boldsymbol{x}, t) \alpha\left(\boldsymbol{x}^{\prime}, t^{\prime}\right)\right\rangle=\int_{-\infty}^{\infty} \frac{d \omega}{2 \pi} \int_{-\infty}^{\infty} \frac{d \omega^{\prime}}{2 \pi} e^{i\left(\omega t-\omega^{\prime} t^{\prime}\right)}\left\langle\alpha(\boldsymbol{x}, \omega) \alpha^{*}\left(\boldsymbol{x}^{\prime}, \omega^{\prime}\right)\right\rangle
$$

For homogeneous, stationary statistics $\left\langle\alpha(\boldsymbol{x}, t) \alpha\left(\boldsymbol{x}^{\prime}, t^{\prime}\right)\right\rangle$ should be a function only of $\boldsymbol{r}=\boldsymbol{x}-\boldsymbol{x}^{\prime}$ and $\tau=t-t^{\prime}$. This immediately tells us that $\left\langle\alpha(\boldsymbol{x}, \omega) \alpha^{*}\left(\boldsymbol{x}^{\prime}, \omega^{\prime}\right)\right\rangle$ must be proportional to a delta function, $\delta\left(\omega-\omega^{\prime}\right)$. That is, we can write

$$
\left\langle\alpha(\boldsymbol{x}, \omega) \alpha^{*}\left(\boldsymbol{x}^{\prime}, \omega^{\prime}\right)\right\rangle=S(\boldsymbol{r}, \omega) 2 \pi \delta\left(\omega-\omega^{\prime}\right),
$$

where $S(\boldsymbol{r}, \omega)$ is the power spectral density, also called the power spectrum. Setting $\boldsymbol{x}=\boldsymbol{x}^{\prime}$ in Eq. (2.8), we see that the autopower spectrum $S(\boldsymbol{r}=0, \omega)$ is real and positive. Inserting Eq. (2.8) into Eq. (2.7), we have

$$
\left\langle\alpha(\boldsymbol{x}, t) \alpha\left(\boldsymbol{x}^{\prime}, t^{\prime}\right)\right\rangle=C(\boldsymbol{r}, \tau)=\int_{-\infty}^{\infty} \frac{d \omega}{2 \pi} e^{i \omega \tau} S(\boldsymbol{r}, \omega)
$$

Eq. (2.9) tells us that the power spectrum $S(\boldsymbol{r}, \omega)$ is equal to the temporal Fourier 
transform of the correlation function $C(\boldsymbol{r}, \tau)$. That is,

$$
S(\boldsymbol{r}, \omega)=\int_{-\infty}^{\infty} d \tau e^{-i \omega \tau} C(\boldsymbol{r}, \tau)
$$

Eqs. (2.9) or (2.10) are known as the Wiener-Khintchin theorem. It follows from Eq. (2.9) that the fluctuation power can be written as

$$
\left\langle\alpha^{2}\right\rangle=C(\boldsymbol{r}=0, \tau=0)=\int_{-\infty}^{\infty} \frac{d \omega}{2 \pi} S(\boldsymbol{r}=0, \omega)
$$

The autopower spectrum is also called the frequency spectrum, since the autopower spectral density $S(\boldsymbol{r}=0, \omega)$ indicates the frequency distribution of the fluctuation energy.

Note that the results of this section can be obtained from Sections 1.3-1.4 by replacing the discrete indices with the spatial coordinates. For instance,

$$
\boldsymbol{C}(\tau) \rightarrow C_{n m}(\tau) \rightarrow C\left(\boldsymbol{x}, \boldsymbol{x}^{\prime}, \tau\right)=C(\boldsymbol{r}, \tau)
$$

and

$$
\boldsymbol{S}(\omega) \rightarrow S_{n m}(\omega) \rightarrow S\left(\boldsymbol{x}, \boldsymbol{x}^{\prime}, \omega\right)=S(\boldsymbol{r}, \omega)
$$

Note that the transpose matrix corresponds to the transpose on $\boldsymbol{x}$ and $\boldsymbol{x}^{\prime}$. For instance,

$$
\boldsymbol{C}^{T}(\tau) \rightarrow C_{m n}(\tau) \rightarrow C\left(\boldsymbol{x}^{\prime}, \boldsymbol{x}, \tau\right)=C(-\boldsymbol{r}, \tau)
$$

and

$$
\boldsymbol{S}^{T}(\omega) \rightarrow S_{m n}(\omega) \rightarrow S\left(\boldsymbol{x}^{\prime}, \boldsymbol{x}, \omega\right)=S(-\boldsymbol{r}, \omega)
$$

Thus Eq. (1.9) implies

$$
C(\boldsymbol{r}, \tau)=C(-\boldsymbol{r},-\tau),
$$


and Eq. (1.23) implies

$$
S(\boldsymbol{r}, \omega)=S(-\boldsymbol{r},-\omega) .
$$

In particular, the autocorrelation function $C(\boldsymbol{r}=0, \tau)$ is even in $\tau$ and the autopower spectrum $S(\boldsymbol{r}=0, \omega)$ is even in $\omega$. Thus it follows from Eqs. (2.9) and (2.10) that

$$
C(\boldsymbol{r}=0, \tau)=\int_{0}^{\infty} \frac{d \omega}{\pi} \cos (\omega \tau) S(\boldsymbol{r}=0, \omega)
$$

and

$$
S(\boldsymbol{r}=0, \omega)=2 \int_{0}^{\infty} d \tau \cos (\omega \tau) C(\boldsymbol{r}=0, \tau) .
$$

Finally, we write Eq. (2.10) as

$$
S(\boldsymbol{r}, \omega)=S_{+}(\boldsymbol{r}, \omega)+S_{-}(\boldsymbol{r}, \omega),
$$

defining

$$
S_{+}(\boldsymbol{r}, \omega)=\int_{0}^{\infty} d \tau e^{-i \omega \tau} C(\boldsymbol{r}, \tau)
$$

and

$$
S_{-}(\boldsymbol{r}, \omega)=\int_{-\infty}^{0} d \tau e^{-i \omega \tau} C(\boldsymbol{r}, \tau) .
$$

Note that Eq. (1.32) implies

$$
S_{-}(\boldsymbol{r}, \omega)=S_{+}(-\boldsymbol{r},-\omega) .
$$

Thus Eq. (2.20) can be rewritten as

$$
S(\boldsymbol{r}, \omega)=S_{+}(\boldsymbol{r}, \omega)+S_{+}(-\boldsymbol{r},-\omega) .
$$

In the case of time-reversal symmetry, Eq. (1.38) implies that the power spectrum 
is given by

$$
S(\boldsymbol{r}, \omega)=S_{+}(\boldsymbol{r}, \omega)+S_{+}^{*}(\boldsymbol{r}, \omega)=2 \operatorname{Re} S_{+}(\boldsymbol{r}, \omega) .
$$

\subsubsection{Wavenumber spectrum}

For systems that are invariant under translations in space, the fluctuations $\alpha(\boldsymbol{x}, t)$ may be written as spatial Fourier integrals

$$
\alpha(\boldsymbol{x}, t)=\int \frac{d^{3} k}{(2 \pi)^{3}} e^{i \boldsymbol{k} \cdot \boldsymbol{x}} \alpha(\boldsymbol{k}, t)
$$

where $\boldsymbol{k}$ is a real wave vector and

$$
\alpha(\boldsymbol{k}, t)=\int d^{3} x e^{-i \boldsymbol{k} \cdot \boldsymbol{x}} \alpha(\boldsymbol{x}, t)
$$

denotes the spatial Fourier transform of $\alpha(\boldsymbol{x}, t)$.

The average of the product of two real quantities $\alpha(\boldsymbol{x}, t)$ and $\alpha\left(\boldsymbol{x}^{\prime}, t^{\prime}\right)$ can be written as

$$
\left\langle\alpha(\boldsymbol{x}, t) \alpha\left(\boldsymbol{x}^{\prime}, t^{\prime}\right)\right\rangle=\int \frac{d^{3} k}{(2 \pi)^{3}} \int \frac{d^{3} k^{\prime}}{(2 \pi)^{3}} e^{i\left(\boldsymbol{k} \cdot \boldsymbol{x}-\boldsymbol{k}^{\prime} \cdot \boldsymbol{x}^{\prime}\right)}\left\langle\alpha(\boldsymbol{k}, t) \alpha^{*}\left(\boldsymbol{k}^{\prime}, t^{\prime}\right)\right\rangle
$$

For homogeneous, stationary statistics $\left\langle\alpha(\boldsymbol{x}, t) \alpha\left(\boldsymbol{x}^{\prime}, t^{\prime}\right)\right\rangle$ should be a function only of $\boldsymbol{r}=\boldsymbol{x}-\boldsymbol{x}^{\prime}$ and $\tau=t-t^{\prime}$. This immediately tells us that $\left\langle\alpha(\boldsymbol{k}, t) \alpha^{*}\left(\boldsymbol{k}^{\prime}, t^{\prime}\right)\right\rangle$ must be proportional to a delta function, $\delta^{3}\left(\boldsymbol{k}-\boldsymbol{k}^{\prime}\right)$. That is, we can write

$$
\left\langle\alpha(\boldsymbol{k}, t) \alpha^{*}\left(\boldsymbol{k}^{\prime}, t^{\prime}\right)\right\rangle=C(\boldsymbol{k}, \tau)(2 \pi)^{3} \delta^{3}\left(\boldsymbol{k}-\boldsymbol{k}^{\prime}\right)
$$

where $C(\boldsymbol{k}, \tau)$ is called the wavenumber spectrum. Setting $t=t^{\prime}$ in Eq. (2.29), we see that $C(\boldsymbol{k}, \tau=0)$ is real and positive:

$$
C^{*}(\boldsymbol{k}, \tau=0)=C(\boldsymbol{k}, \tau=0) .
$$


It follows from Eq. (2.16) that $C(\boldsymbol{r}, \tau=0)$ is even in $\boldsymbol{r}$ :

$$
C(\boldsymbol{r}, \tau=0)=C(-\boldsymbol{r}, \tau=0)
$$

The spatial Fourier transform of Eq. (2.31) gives

$$
C(\boldsymbol{k}, \tau=0)=C(-\boldsymbol{k}, \tau=0)
$$

Thus the real quantity $C(\boldsymbol{k}, \tau=0)$ is even in $\boldsymbol{k}$.

Inserting Eq. (2.29) into Eq. (2.28), we have

$$
\left\langle\alpha(\boldsymbol{x}, t) \alpha\left(\boldsymbol{x}^{\prime}, t^{\prime}\right)\right\rangle=C(\boldsymbol{r}, \tau)=\int \frac{d^{3} k}{(2 \pi)^{3}} e^{i \boldsymbol{k} \cdot \boldsymbol{r}} C(\boldsymbol{k}, \tau)
$$

Eq. (2.33) tells us that $C(\boldsymbol{k}, \tau)$ is equal to the spatial Fourier transform of the correlation function $C(\boldsymbol{r}, \tau)$. That is,

$$
C(\boldsymbol{k}, \tau)=\int d^{3} r e^{-i \boldsymbol{k} \cdot \boldsymbol{r}} C(\boldsymbol{r}, \tau)
$$

It follows from Eq. (2.33) that the fluctuation power can be written as

$$
\left\langle\alpha^{2}\right\rangle=C(\boldsymbol{r}=0, \tau=0)=\int \frac{d^{3} k}{(2 \pi)^{3}} C(\boldsymbol{k}, \tau=0)
$$

\subsubsection{Wavenumber-frequency spectrum}

For translationally invariant systems, the fluctuations $\alpha(\boldsymbol{x}, t)$ may be written as Fourier integrals

$$
\alpha(\boldsymbol{x}, t)=\int \frac{d^{3} k}{(2 \pi)^{3}} e^{i \boldsymbol{k} \cdot \boldsymbol{x}} \int_{-\infty}^{\infty} \frac{d \omega}{2 \pi} e^{i \omega t} \alpha(\boldsymbol{k}, \omega)
$$


where $\omega=2 \pi f$ is a real frequency, $\boldsymbol{k}$ is a real wave vector, and

$$
\alpha(\boldsymbol{k}, \omega)=\int d^{3} x e^{-i \boldsymbol{k} \cdot \boldsymbol{x}} \int_{-\infty}^{\infty} d t e^{-i \omega t} \alpha(\boldsymbol{x}, t)
$$

denotes the spatial and temporal Fourier transforms of $\alpha(\boldsymbol{x}, t)$.

The average of the product of two real quantities $\alpha(\boldsymbol{x}, t)$ and $\alpha\left(\boldsymbol{x}^{\prime}, t^{\prime}\right)$ can be written as

$$
\begin{aligned}
\left\langle\alpha(\boldsymbol{x}, t) \alpha\left(\boldsymbol{x}^{\prime}, t^{\prime}\right)\right\rangle=\quad & \int \frac{d^{3} k}{(2 \pi)^{3}} \int \frac{d^{3} k^{\prime}}{(2 \pi)^{3}} e^{i\left(\boldsymbol{k} \cdot \boldsymbol{x}-\boldsymbol{k}^{\prime} \cdot \boldsymbol{x}^{\prime}\right)} \int_{-\infty}^{\infty} \frac{d \omega}{2 \pi} \int_{-\infty}^{\infty} \frac{d \omega^{\prime}}{2 \pi} e^{i\left(\omega t-\omega^{\prime} t^{\prime}\right)} \\
& \times\left\langle\alpha(\boldsymbol{k}, \omega) \alpha^{*}\left(\boldsymbol{k}^{\prime}, \omega^{\prime}\right)\right\rangle
\end{aligned}
$$

For homogeneous, stationary statistics $\left\langle\alpha(\boldsymbol{x}, t) \alpha\left(\boldsymbol{x}^{\prime}, t^{\prime}\right)\right\rangle$ should be a function only of $\boldsymbol{r}=\boldsymbol{x}-\boldsymbol{x}^{\prime}$ and $\tau=t-t^{\prime}$. This immediately tells us that $\left\langle\alpha(\boldsymbol{k}, \omega) \alpha^{*}\left(\boldsymbol{k}^{\prime}, \omega^{\prime}\right)\right\rangle$ must be proportional to delta functions, $\delta^{3}\left(\boldsymbol{k}-\boldsymbol{k}^{\prime}\right) \delta\left(\omega-\omega^{\prime}\right)$. That is, we can write

$$
\left\langle\alpha(\boldsymbol{k}, \omega) \alpha^{*}\left(\boldsymbol{k}^{\prime}, \omega^{\prime}\right)\right\rangle=S(\boldsymbol{k}, \omega)(2 \pi)^{3} \delta^{3}\left(\boldsymbol{k}-\boldsymbol{k}^{\prime}\right) 2 \pi \delta\left(\omega-\omega^{\prime}\right),
$$

where $S(\boldsymbol{k}, \omega)$ is called the wavenumber-frequency spectrum. Since the delta functions set $\boldsymbol{k}=\boldsymbol{k}^{\prime}$ and $\omega=\omega^{\prime}$ in Eq. (2.39), we see that the statistical quantity $S(\boldsymbol{k}, \omega)$ is real and positive:

$$
S^{*}(\boldsymbol{k}, \omega)=S(\boldsymbol{k}, \omega)
$$

Inserting Eq. (2.39) into Eq. (2.38), we have

$$
\left\langle\alpha(\boldsymbol{x}, t) \alpha\left(\boldsymbol{x}^{\prime}, t^{\prime}\right)\right\rangle=C(\boldsymbol{r}, \tau)=\int_{-\infty}^{\infty} \frac{d \omega}{2 \pi} e^{i \omega \tau} \int \frac{d^{3} k}{(2 \pi)^{3}} e^{i \boldsymbol{k} \cdot \boldsymbol{r}} S(\boldsymbol{k}, \omega) .
$$

Eq. (2.41) tells us that $S(\boldsymbol{k}, \omega)$ is equal to the spatial and temporal Fourier trans- 
forms of the correlation function $C(\boldsymbol{r}, \tau)$. That is,

$$
S(\boldsymbol{k}, \omega)=\int_{-\infty}^{\infty} d \tau e^{-i \omega \tau} \int d^{3} r e^{-i \boldsymbol{k} \cdot \boldsymbol{r}} C(\boldsymbol{r}, \tau)
$$

Since the correlation function is real, Eq. (2.42) shows that

$$
S^{*}(\boldsymbol{k}, \omega)=S(-\boldsymbol{k},-\omega) .
$$

Then Eq. (2.40) tells us that

$$
S(\boldsymbol{k}, \omega)=S(-\boldsymbol{k},-\omega) .
$$

It follows from Eq. (2.41) that the fluctuation power can be written as

$$
\left\langle\alpha^{2}\right\rangle=C(\boldsymbol{r}=0, \tau=0)=\int \frac{d^{3} k}{(2 \pi)^{3}} \int_{-\infty}^{\infty} \frac{d \omega}{2 \pi} S(\boldsymbol{k}, \omega) .
$$

Comparing Eq. (2.9) with Eq. (2.41), we see that

$$
S(\boldsymbol{r}, \omega)=\int \frac{d^{3} k}{(2 \pi)^{3}} e^{i \boldsymbol{k} \cdot \boldsymbol{r}} S(\boldsymbol{k}, \omega) .
$$

That is, $S(\boldsymbol{k}, \omega)$ is equal to the spatial Fourier transform of the power spectrum $S(\boldsymbol{r}, \omega)$. In particular, the autopower spectrum is given by

$$
S(\boldsymbol{r}=0, \omega)=\int \frac{d^{3} k}{(2 \pi)^{3}} S(\boldsymbol{k}, \omega)
$$

Comparing Eq. (2.33) with Eq. (2.41), we find that

$$
C(\boldsymbol{k}, \tau)=\int_{-\infty}^{\infty} \frac{d \omega}{2 \pi} e^{i \omega \tau} S(\boldsymbol{k}, \omega)
$$

That is, $S(\boldsymbol{k}, \omega)$ is equal to the temporal Fourier transform of $C(\boldsymbol{k}, \tau)$. Note that 
Eq. (2.48) is also the spatial Fourier transform of the Wiener-Khintchin theorem Eq. (2.9). It follows from Eq. (2.48) that $S(\boldsymbol{k}, \omega)$ must satisfy the condition

$$
C(\boldsymbol{k}, \tau=0)=\int_{-\infty}^{\infty} \frac{d \omega}{2 \pi} S(\boldsymbol{k}, \omega)
$$

Finally, we write the spatial Fourier transform of Eq. (2.20) as

$$
S(\boldsymbol{k}, \omega)=S_{+}(\boldsymbol{k}, \omega)+S_{-}(\boldsymbol{k}, \omega)
$$

where $S_{+}(\boldsymbol{k}, \omega)$ and $S_{-}(\boldsymbol{k}, \omega)$ are the spatial Fourier transforms of $S_{+}(\boldsymbol{r}, \omega)$ and $S_{-}(\boldsymbol{r}, \omega)$, respectively. For instance,

$$
S_{+}(\boldsymbol{r}, \omega)=\int \frac{d^{3} k}{(2 \pi)^{3}} e^{i \boldsymbol{k} \cdot \boldsymbol{r}} S_{+}(\boldsymbol{k}, \omega)
$$

and

$$
S_{+}(\boldsymbol{k}, \omega)=\int d^{3} r e^{-i \boldsymbol{k} \cdot \boldsymbol{r}} \int_{0}^{\infty} d \tau e^{-i \omega \tau} C(\boldsymbol{r}, \tau) .
$$

The spatial Fourier transform of Eq. (2.23) gives

$$
S_{-}(\boldsymbol{k}, \omega)=S_{+}(-\boldsymbol{k},-\omega)=S_{+}^{*}(\boldsymbol{k}, \omega)
$$

Thus Eq. (2.50) can be rewritten as

$$
S(\boldsymbol{k}, \omega)=S_{+}(\boldsymbol{k}, \omega)+S_{+}^{*}(\boldsymbol{k}, \omega)=2 \operatorname{Re} S_{+}(\boldsymbol{k}, \omega) .
$$

Note that this expression satisfies the condition Eq. (2.40). Inserting Eq. (2.54) into Eq. (2.47), we find that the autopower spectrum

$$
S(\boldsymbol{r}=0, \omega)=2 \operatorname{Re} \int \frac{d^{3} k}{(2 \pi)^{3}} S_{+}(\boldsymbol{k}, \omega) .
$$




\subsection{Isotropic statistics}

For isotropic statistics, probability distributions are invariant under rotations in space, so that the statistical properties of isotropic fluctuations are the same in all directions. For instance, the spatial correlations between two points can only depend on the separation distance $r=|\boldsymbol{r}|$, and the corresponding spatial Fourier transforms can only depend on the magnitude $k=|\boldsymbol{k}|$ of the real wave vector.

Thus for isotropic statistics, the correlation function: $C( \pm \boldsymbol{r}, \tau)=C(r, \tau)$, the spatial Fourier transform of the correlation function: $C( \pm \boldsymbol{k}, \tau)=C(k, \tau)$, the power spectrum: $S( \pm \boldsymbol{r}, \omega)=S(r, \omega)$, and the spatial Fourier transform of the power spectrum: $S( \pm \boldsymbol{k}, \omega)=S(k, \omega)$. Then Eq. (2.16) becomes

$$
C(r, \tau)=C(r,-\tau)
$$

This immediately tells us that isotropic fluctuations have time-reversal symmetry. In the case of spatial isotropy, Eq. (2.25) becomes

$$
S(r, \omega)=2 \operatorname{Re} S_{+}(r, \omega) .
$$

\subsection{Energy spectrum}

For isotropic statistics, the fluctuation power Eq. (2.35) can be written as

$$
\left\langle\alpha^{2}\right\rangle=C(r=0, \tau=0)=\int \frac{d^{3} k}{(2 \pi)^{3}} C(k, \tau=0)=\int_{0}^{\infty} d k E(k)
$$

where $E(k)$ is called the energy spectrum. It is clear that the energy spectrum indicates the distribution of the fluctuation energy over different length scales, and $E(k) d k$ specifies the fluctuation power in the wave number interval $d k$.

The wave number dependence of $E(k)$ may be inferred from $C(k, \tau=0)$ as 
follows. In three dimensions, Eq. (2.58) becomes

$$
\left\langle\alpha^{2}\right\rangle=\int_{0}^{\infty} \frac{4 \pi k^{2} d k}{(2 \pi)^{3}} C(k, \tau=0)=\int_{0}^{\infty} d k E(k)
$$

so the three-dimensional energy spectrum is defined by

$$
E(k)=\frac{4 \pi k^{2}}{(2 \pi)^{3}} C(k, \tau=0)=\frac{k^{2}}{2 \pi^{2}} C(k, \tau=0) .
$$

In two dimensions, Eq. (2.58) becomes

$$
\left\langle\alpha^{2}\right\rangle=\int_{0}^{\infty} \frac{2 \pi k d k}{(2 \pi)^{2}} C(k, \tau=0)=\int_{0}^{\infty} d k E(k)
$$

so the two-dimensional energy spectrum is defined by

$$
E(k)=\frac{2 \pi k}{(2 \pi)^{2}} C(k, \tau=0)=\frac{k}{2 \pi} C(k, \tau=0) .
$$

In one dimension, Eq. (2.58) becomes

$$
\left\langle\alpha^{2}\right\rangle=\int_{-\infty}^{\infty} \frac{d k}{2 \pi} C(|k|, \tau=0)=\int_{0}^{\infty} d k E(k) .
$$

Since isotropy requires $C(k, \tau=0)$ to be even in $k$, the one-dimensional energy spectrum is defined by

$$
E(k)=\frac{2}{2 \pi} C(k, \tau=0)=\frac{1}{\pi} C(k, \tau=0) .
$$

\subsection{Markovian noise spectrum}

To obtain the spatially homogeneous version of Markovian noise spectra in Section 1.5 for a single fluctuating quantity, we simply replace the discrete index $n$ by the spatial coordinate $\boldsymbol{x}$, and write the matrix products as convolution integrals. 
For instance, the unit matrix

$$
\boldsymbol{I} \rightarrow \delta_{n m} \rightarrow \delta\left(\boldsymbol{x}-\boldsymbol{x}^{\prime}\right)
$$

and the response matrix

$$
\boldsymbol{\Lambda} \rightarrow \Lambda_{n m} \rightarrow \Lambda\left(\boldsymbol{x}, \boldsymbol{x}^{\prime}\right)=\Lambda(\boldsymbol{r})
$$

Recall that the transpose matrix corresponds to the transpose on $\boldsymbol{x}$ and $\boldsymbol{x}^{\prime}$, so

$$
\Lambda^{T} \rightarrow \Lambda_{m n} \rightarrow \Lambda\left(\boldsymbol{x}^{\prime}, \boldsymbol{x}\right)=\Lambda(-\boldsymbol{r})
$$

We denote the spatial Fourier transform of $\Lambda(\boldsymbol{r})$ by

$$
\Lambda(\boldsymbol{k})=\int d^{3} r e^{-i \boldsymbol{k} \cdot \boldsymbol{r}} \Lambda(\boldsymbol{r})=\int d^{3} r[\cos (\boldsymbol{k} \cdot \boldsymbol{r})-i \sin (\boldsymbol{k} \cdot \boldsymbol{r})] \Lambda(\boldsymbol{r})
$$

Since $\Lambda(\boldsymbol{r})$ is real, Eq. (2.68) shows that the real part of $\Lambda(\boldsymbol{k})$ is even in $\boldsymbol{k}$ and the imaginary part of $\Lambda(\boldsymbol{k})$ is odd in $\boldsymbol{k}$.

Using Eqs. (2.65) and (2.66), we can express the spatially homogeneous version of Eq. (1.56) as

$$
\int d^{3} x^{\prime \prime}\left[\Lambda\left(\boldsymbol{x}-\boldsymbol{x}^{\prime \prime}\right)+i \omega \delta\left(\boldsymbol{x}-\boldsymbol{x}^{\prime \prime}\right)\right] S_{+}\left(\boldsymbol{x}^{\prime \prime}-\boldsymbol{x}^{\prime}, \omega\right)=C\left(\boldsymbol{x}-\boldsymbol{x}^{\prime}, \tau=0\right)
$$

Then the spatial Fourier transform of the convolution integral Eq. (2.69) gives

$$
[\Lambda(\boldsymbol{k})+i \omega] S_{+}(\boldsymbol{k}, \omega)=C(\boldsymbol{k}, \tau=0)
$$

or

$$
S_{+}(\boldsymbol{k}, \omega)=\frac{C(\boldsymbol{k}, \tau=0)}{\Lambda(\boldsymbol{k})+i \omega}
$$


Similarly, using Eq. (2.67) we can express the spatially homogeneous version of Eq. (1.58) as

$$
\int d^{3} x^{\prime \prime} S_{-}\left(\boldsymbol{x}-\boldsymbol{x}^{\prime \prime}, \omega\right)\left[\Lambda\left(\boldsymbol{x}^{\prime}-\boldsymbol{x}^{\prime \prime}\right)-i \omega \delta\left(\boldsymbol{x}^{\prime \prime}-\boldsymbol{x}^{\prime}\right)\right]=C\left(\boldsymbol{x}-\boldsymbol{x}^{\prime}, \tau=0\right)
$$

The Fourier transform of $\Lambda(-\boldsymbol{r})$ is $\Lambda(-\boldsymbol{k})$, so the spatial Fourier transform of the convolution integral Eq. (2.71) gives

$$
S_{-}(\boldsymbol{k}, \omega)[\Lambda(-\boldsymbol{k})-i \omega]=C(\boldsymbol{k}, \tau=0)
$$

or

$$
S_{-}(\boldsymbol{k}, \omega)=\frac{C(\boldsymbol{k}, \tau=0)}{\Lambda(-\boldsymbol{k})-i \omega} .
$$

Since $C(\boldsymbol{k}, \tau=0)$ is a real, even function of $\boldsymbol{k}$ and $\Lambda(-\boldsymbol{k})=\Lambda^{*}(\boldsymbol{k})$, we confirm that the Markovian spectra Eqs. (2.70) and (2.72) satisfy the condition Eq. (2.53).

Inserting Eq. (2.70) into Eq. (2.54), we have

$$
S(\boldsymbol{k}, \omega)=2 \operatorname{Re} S_{+}(\boldsymbol{k}, \omega)=2 \operatorname{Re}\left[\frac{C(\boldsymbol{k}, \tau=0)}{\Lambda(\boldsymbol{k})+i \omega}\right] .
$$

Thus $S(\boldsymbol{k}, \omega)$ is known in terms of $\Lambda(\boldsymbol{k})$ and $C(\boldsymbol{k}, \tau=0)$. In general, $\Lambda(\boldsymbol{k})$ is complex:

$$
\Lambda(\boldsymbol{k})=\Lambda_{r}(\boldsymbol{k})-i \Lambda_{i}(\boldsymbol{k})
$$

Thus Eq. (2.70) can be rewritten as

$$
S_{+}(\boldsymbol{k}, \omega)=\frac{C(\boldsymbol{k}, \tau=0)}{\Lambda_{r}+i\left(\omega-\Lambda_{i}\right)} .
$$

Using Eq. (2.73) we then obtain

$$
S(\boldsymbol{k}, \omega)=2 \operatorname{Re} S_{+}(\boldsymbol{k}, \omega)=C(\boldsymbol{k}, \tau=0) \frac{2 \Lambda_{r}}{\Lambda_{r}^{2}+\left(\omega-\Lambda_{i}\right)^{2}}
$$


Since $\Lambda_{r}(\boldsymbol{k})$ is even in $\boldsymbol{k}$ and $\Lambda_{i}(\boldsymbol{k})$ is odd in $\boldsymbol{k}$, we confirm that the formula Eq. (2.76) satisfies the condition Eq. (2.44). It follows from Eqs. (2.55) and (2.47) that

$$
S(\boldsymbol{r}=0, \omega)=2 \operatorname{Re} \int \frac{d^{3} k}{(2 \pi)^{3}} S_{+}(\boldsymbol{k}, \omega)=2 \operatorname{Re} \int \frac{d^{3} k}{(2 \pi)^{3}} \frac{C(\boldsymbol{k}, \tau=0)}{\Lambda_{r}+i\left(\omega-\Lambda_{i}\right)}
$$

or

$$
S(\boldsymbol{r}=0, \omega)=\int \frac{d^{3} k}{(2 \pi)^{3}} S(\boldsymbol{k}, \omega)=\int \frac{d^{3} k}{(2 \pi)^{3}} C(\boldsymbol{k}, \tau=0) \frac{2 \Lambda_{r}}{\Lambda_{r}^{2}+\left(\omega-\Lambda_{i}\right)^{2}} .
$$

These are the essential tools of our theory. From Eqs. (2.76) and (2.78) we see that the real part of $\Lambda(\boldsymbol{k})$ is essential for a nonvanishing spectrum and the imaginary part of $\Lambda(\boldsymbol{k})$ shifts the maximum of the spectra away from zero frequency. In the following chapters, we will use some physical models to find out the form of $\Lambda(\boldsymbol{k})$ and $C(\boldsymbol{k}, \tau=0)$, then use Eq. (2.78) to calculate the autopower spectrum. 


\section{Chapter 3}

\section{Diffusion Model}

In this chapter, we will use a diffusion equation to describe the relaxation of homogeneous isotropic fluctuations. To calculate the temporal and spatial correlations due to the diffusion, we will consider two models for the spatial correlations at one instant of time: the delta function and the exponential decay. Finally, we will discuss the oscillation and decay of random fluctuations.

\subsection{Diffusion equation}

For physical systems with diffusion response to random fluctuations, the relaxation of the conditional mean is modeled by the diffusion equation

$$
\frac{\partial}{\partial t}\langle\alpha(\boldsymbol{x}, t)\rangle_{0}=D \nabla^{2}\langle\alpha(\boldsymbol{x}, t)\rangle_{0}
$$

where $D$ is the effective diffusion coefficient of random fluctuations. Comparing Eq. (3.1) with Eq. (2.2), we obtain the diffusion response function

$$
\Lambda\left(\boldsymbol{x}-\boldsymbol{x}^{\prime}\right)=-D \nabla^{2} \delta\left(\boldsymbol{x}-\boldsymbol{x}^{\prime}\right) \quad \text { or } \quad \Lambda(\boldsymbol{r})=-D \nabla^{2} \delta(\boldsymbol{r})
$$


The spatial Fourier transform of Eq. (3.2) gives

$$
\Lambda(k)=D k^{2},
$$

where $k=|\boldsymbol{k}|$ is the magnitude of the wave vector.

Consider stationary Markovian random fluctuations that are statistically homogeneous and isotropic. Inserting Eq. (3.3) into Eq. (2.70), we have

$$
S_{+}(k, \omega)=\frac{C(k, \tau=0)}{D k^{2}+i \omega}
$$

Then Eq. (2.73) gives

$$
S(k, \omega)=2 \operatorname{Re} S_{+}(k, \omega)=C(k, \tau=0) \frac{2 D k^{2}}{\left(D k^{2}\right)^{2}+\omega^{2}} .
$$

Thus the frequency dependence of $S(k, \omega)$ takes the Lorentzian form. Since

$$
\int_{-\infty}^{\infty} \frac{d \omega}{2 \pi} \frac{2 D k^{2}}{\left(D k^{2}\right)^{2}+\omega^{2}}=1
$$

we confirm that the Lorentzian form of $S(k, \omega)$ satisfies the condition Eq. (2.49).

Inserting Eq. (3.5) into Eq. (2.47), we have

$$
S(r=0, \omega)=\int \frac{d^{3} k}{(2 \pi)^{3}} C(k, \tau=0) \frac{2 D k^{2}}{D^{2} k^{4}+\omega^{2}}
$$

From Eq. (2.58) we see that for isotropic statistics, Eq. (3.7) can be rewritten as

$$
S(r=0, \omega)=\int_{0}^{\infty} d k E(k) \frac{2 D k^{2}}{D^{2} k^{4}+\omega^{2}}
$$

In view of Eqs. (2.58) and (3.6), we confirm that the formulae Eqs. (3.7) and (3.8) for the autopower spectrum satisfy the condition Eq. (2.11). 
Inserting Eq. (3.5) into Eq. (2.48) we obtain

$$
C(k, \tau) / C(k, \tau=0)=\int_{-\infty}^{\infty} \frac{d \omega}{2 \pi} e^{i \omega \tau} \frac{2 D k^{2}}{\left(D k^{2}\right)^{2}+\omega^{2}}=e^{-k^{2} D|\tau|}
$$

Thus the wave number dependence of $C(k, \tau)$ takes the Gaussian form.

\subsection{Delta function model}

For simplicity, let us consider the one-dimensional fluctuations, in the sense that the correlations only vary in one dimension. We assume that for isotropic statistics, the spatial correlations at one instant of time take the exponential decay form

$$
\left\langle\alpha(x, t) \alpha\left(x^{\prime}, t\right)\right\rangle=C(r, \tau=0)=\left\langle\alpha^{2}\right\rangle e^{-|r| / \lambda_{c}},
$$

where $r=x-x^{\prime}$ is the spatial coordinate difference, $\lambda_{c}$ is the decay length, and $\left\langle\alpha^{2}\right\rangle$ is the mean square value of the fluctuations.

The correlation length, also known as the integral length scale, is defined by

$$
L_{c}=\int_{0}^{\infty} d r C(r, \tau=0) /\left\langle\alpha^{2}\right\rangle
$$

That is, the area under the spatial correlation function at a single time is equal to the mean square deviations $\left\langle\alpha^{2}\right\rangle$ times the correlation length $L_{c}$.

The correlation length of the exponential decay Eq. (3.10) is given by

$$
L_{c}=\int_{0}^{\infty} d r e^{-r / \lambda_{c}}=\lambda_{c}
$$

Thus the correlation length of an exponential decay is the decay length $\lambda_{c}$. Since

$$
\lim _{\lambda_{c} \rightarrow 0} \frac{e^{-|r| / \lambda_{c}}}{2 \lambda_{c}}=\delta(r)
$$


for the correlation length $\lambda_{c} \rightarrow 0$, Eq. (3.10) becomes

$$
\left\langle\alpha(x, t) \alpha\left(x^{\prime}, t\right)\right\rangle=C(r, \tau=0)=\left\langle\alpha^{2}\right\rangle 2 \lambda_{c} \delta(r) .
$$

Eq. (3.14) states that at a given instant of time the fluctuations at different spatial points are uncorrelated. The Fourier transform of Eq. (3.14) gives

$$
C(k, \tau=0)=\int_{-\infty}^{\infty} d r e^{-i k r} C(r, \tau=0)=\left\langle\alpha^{2}\right\rangle 2 \lambda_{c}
$$

To demonstrate the general features of the spatial and temporal correlations due to the diffusion, we will use the delta function Eq. (3.14) for the spatial correlations at one instant of time in this section.

With $C(k, \tau=0)$ given by Eq. (3.15), it follows from Eqs. (3.4) and (3.5) that

$$
S_{+}(k, \omega)=\left\langle\alpha^{2}\right\rangle \frac{2 \lambda_{c}}{D k^{2}+i \omega}
$$

and

$$
S(k, \omega)=\left\langle\alpha^{2}\right\rangle 2 \lambda_{c} \frac{2 D k^{2}}{\left(D k^{2}\right)^{2}+\omega^{2}} .
$$

Since

$$
\lim _{k \rightarrow 0} \frac{D k^{2}}{\left(D k^{2}\right)^{2}+\omega^{2}}=\pi \delta(\omega)
$$

for wave numbers $k \rightarrow 0, S(k, \omega)$ becomes divergent at zero frequency.

The correlations at one spatial point can be calculated as follows. In one dimension, Eq. (2.55) becomes

$$
S(r=0, \omega)=2 \operatorname{Re} \int_{-\infty}^{\infty} \frac{d k}{2 \pi} S_{+}(k, \omega) .
$$


With $S_{+}(k, \omega)$ given by Eq. (3.16), the contour integration of Eq. (3.19) gives

$$
S(r=0, \omega) /\left\langle\alpha^{2}\right\rangle=2 \operatorname{Re} \int_{-\infty}^{\infty} \frac{d k}{2 \pi} \frac{2 \lambda_{c}}{D k^{2}+i \omega}=\frac{2 \lambda_{c}}{\sqrt{2 D|\omega|}}
$$

Thus the autopower spectrum decays as the frequency increases.

According to the Wiener-Khintchin theorem, the autocorrelation function is the inverse Fourier transform of the autopower spectrum. Inserting Eq. (3.20) into Eq. (2.18), we have

$$
C(r=0, \tau) /\left\langle\alpha^{2}\right\rangle=\int_{0}^{\infty} \frac{d \omega}{\pi} \cos (\omega \tau) \frac{2 \lambda_{c}}{\sqrt{2 D|\omega|}}=\frac{2 \lambda_{c}}{\sqrt{\pi 4 D|\tau|}}
$$

Thus the autocorrelation function decays as the separation time $|\tau|$ increases.

The correlations between two spatial points can be calculated as follows. In one dimension, Eq. (2.33) becomes

$$
\left\langle\alpha(x, t) \alpha\left(x^{\prime}, t^{\prime}\right)\right\rangle=C(r, \tau)=\int_{-\infty}^{\infty} \frac{d k}{2 \pi} e^{i k r} C(k, \tau)
$$

Inserting Eq. (3.15) into Eq. (3.9), we have

$$
C(k, \tau)=\left\langle\alpha^{2}\right\rangle 2 \lambda_{c} e^{-k^{2} D|\tau|} .
$$

Then the integration of Eq. (3.22) gives

$$
C(r, \tau)=\left\langle\alpha^{2}\right\rangle \frac{2 \lambda_{c}}{\sqrt{\pi 4 D|\tau|}} \exp \left(\frac{-r^{2}}{4 D|\tau|}\right)
$$

Thus the correlation function decays as the separation distance $|r|$ increases. Setting $r=0$ in Eq. (3.24), we again obtain the autocorrelation function Eq. (3.21). It is clear that the fluctuations at different spatial points become correlated due to the 
finite separation time and the diffusion. Since

$$
\lim _{\tau \rightarrow 0} \frac{1}{\sqrt{\pi 4 D|\tau|}} \exp \left(\frac{-r^{2}}{4 D|\tau|}\right)=\delta(r)
$$

for $\tau \rightarrow$ 0, Eq. (3.24) reduces to the spatial correlation function at one instant of time Eq. (3.14).

In one dimension, Eq. (2.51) becomes

$$
S_{+}(r, \omega)=\int_{-\infty}^{\infty} \frac{d k}{2 \pi} e^{i k r} S_{+}(k, \omega)
$$

and Eq. (2.25) becomes

$$
S(r, \omega)=2 \operatorname{Re} S_{+}(r, \omega) .
$$

With $S_{+}(k, \omega)$ given by Eq. (3.16), we obtain

$$
S(r, \omega)=\left\langle\alpha^{2}\right\rangle \frac{2 \lambda_{c}}{\sqrt{2 D|\omega|}} \exp \left(-|r| \sqrt{\frac{|\omega|}{2 D}}\right) \sqrt{2} \cos \left(|r| \sqrt{\frac{|\omega|}{2 D}}+\frac{\pi}{4}\right)
$$

Thus the power spectrum decays as the separation distance $|r|$ increases. Setting $r=0$ in Eq. (3.28), we again obtain the autopower spectrum Eq. (3.20).

In summary, we have found that the diffusion process can give rise to the decay of the correlation function and a broad frequency spectrum of random fluctuations.

\subsection{Exponential decay model}

The plasma theory and experiments show that the spatial correlations can be well approximated by an exponential decay $[11,14,15,16]$. Hence to study the physical systems that have finite correlation length, we assume that the spatial correlations 
of isotropic fluctuations at one instant of time take the form

$$
\left\langle\alpha(\boldsymbol{x}, t) \alpha\left(\boldsymbol{x}^{\prime}, t\right)\right\rangle=C(\boldsymbol{r}, \tau=0)=\left\langle\alpha^{2}\right\rangle e^{-|\boldsymbol{r}| / \lambda_{c}},
$$

where $\lambda_{c}$ is the correlation length and $\left\langle\alpha^{2}\right\rangle$ is the mean square value of the fluctuations. Note that the slope discontinuity at the origin reflects inability of the exponential decay to resolve fine structure at length scales that are much shorter than the correlation length [11]. From now on we consider the exponential decay for the spatial correlations at one instant of time.

\subsection{Energy spectrum of exponential decay}

The exponential decay form Eq. (3.29) holds for all dimensions, but its corresponding spatial Fourier transforms depend on the spatial dimension.

In one dimension, Eq. (2.34) becomes

$$
C(k, \tau=0)=\int_{-\infty}^{\infty} d r e^{-i k r} C(|r|, \tau=0)
$$

Inserting Eq. (3.29) into Eq. (3.30), we have

$$
C(|k|, \tau=0) /\left\langle\alpha^{2}\right\rangle=2 \int_{0}^{\infty} d r e^{-r / \lambda_{c}} \cos (k r)=\frac{2 \lambda_{c}}{1+k^{2} \lambda_{c}^{2}}
$$

Using Eq. (2.64), we then obtain the one-dimensional energy spectrum

$$
E(k)=\frac{1}{\pi} C(k, \tau=0)=\left\langle\alpha^{2}\right\rangle \frac{2}{\pi} \frac{\lambda_{c}}{1+k^{2} \lambda_{c}^{2}} .
$$

In two dimensions, Eq. (2.34) becomes

$$
C(k, \tau=0)=\int d^{2} r e^{-i \boldsymbol{k} \cdot \boldsymbol{r}} C(r, \tau=0)
$$


Inserting Eq. (3.29) into Eq. (3.33), we have

$$
C(k, \tau=0) /\left\langle\alpha^{2}\right\rangle=\int_{0}^{\infty} r d r e^{-r / \lambda_{c}} \int_{0}^{2 \pi} d \theta e^{-i k r \cos \theta}=\frac{2 \pi \lambda_{c}^{2}}{\left(1+k^{2} \lambda_{c}^{2}\right)^{3 / 2}}
$$

Using Eq. (2.62), we then obtain the two-dimensional energy spectrum

$$
E(k)=\frac{k}{2 \pi} C(k, \tau=0)=\left\langle\alpha^{2}\right\rangle \frac{k \lambda_{c}^{2}}{\left(1+k^{2} \lambda_{c}^{2}\right)^{3 / 2}} .
$$

In three dimensions, Eq. (2.34) becomes

$$
C(k, \tau=0)=\int d^{3} r e^{-i \boldsymbol{k} \cdot \boldsymbol{r}} C(r, \tau=0)
$$

Inserting Eq. (3.29) into Eq. (3.36), we have

$$
C(k, \tau=0) /\left\langle\alpha^{2}\right\rangle=\int_{0}^{\infty} 4 \pi r^{2} d r \frac{\sin (k r)}{k r} e^{-r / \lambda_{c}}=\frac{8 \pi \lambda_{c}^{3}}{\left(1+k^{2} \lambda_{c}^{2}\right)^{2}}
$$

Using Eq. (2.60), we then obtain the three-dimensional energy spectrum

$$
E(k)=\frac{k^{2}}{2 \pi^{2}} C(k, \tau=0)=\left\langle\alpha^{2}\right\rangle \frac{4}{\pi} \frac{k^{2} \lambda_{c}^{3}}{\left(1+k^{2} \lambda_{c}^{2}\right)^{2}} .
$$

The linear and log-log plots of normalized energy spectra are shown in Figs. 3.1 and 3.2, respectively. From Fig. 3.2, we see that the energy spectra of the exponential decay have the following key features. At small wave numbers $k \lambda_{c}<1$, the 1-d energy spectrum Eq. (3.32) becomes flat, but the 3-d energy spectrum Eq. (3.38) drops to zero. At large wave numbers $k \lambda_{c}>2 \pi$, the energy spectra become parallel to each other, and the 1-d energy spectrum is on the left side of the 3-d energy spectrum. And the 2-d energy spectrum Eq. (3.35) is in between of the 1-d and 3-d energy spectra.

In the large wave number range, the wave number dependence of $E(k)$ can 


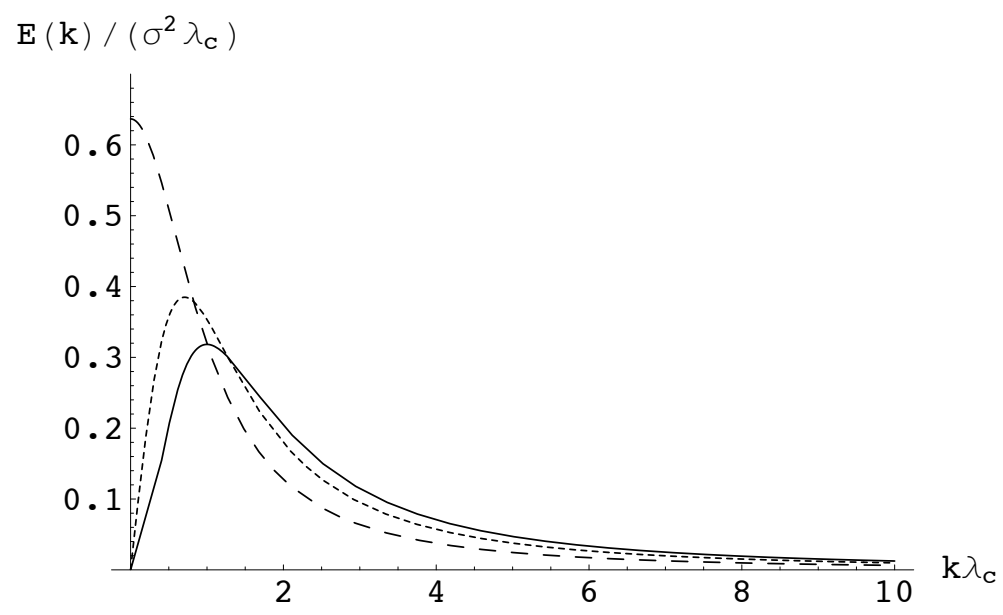

Figure 3.1: Comparison of normalized energy spectra $E(k) /\left(\sigma^{2} \lambda_{c}\right)$ in one dimension (dashed line), two dimensions (dotted line), and three dimensions (black solid line). The energy spectrum $E(k)$ is normalized by mean square deviations $\sigma^{2}=\left\langle\alpha^{2}\right\rangle$ and the correlation length $\lambda_{c}$.

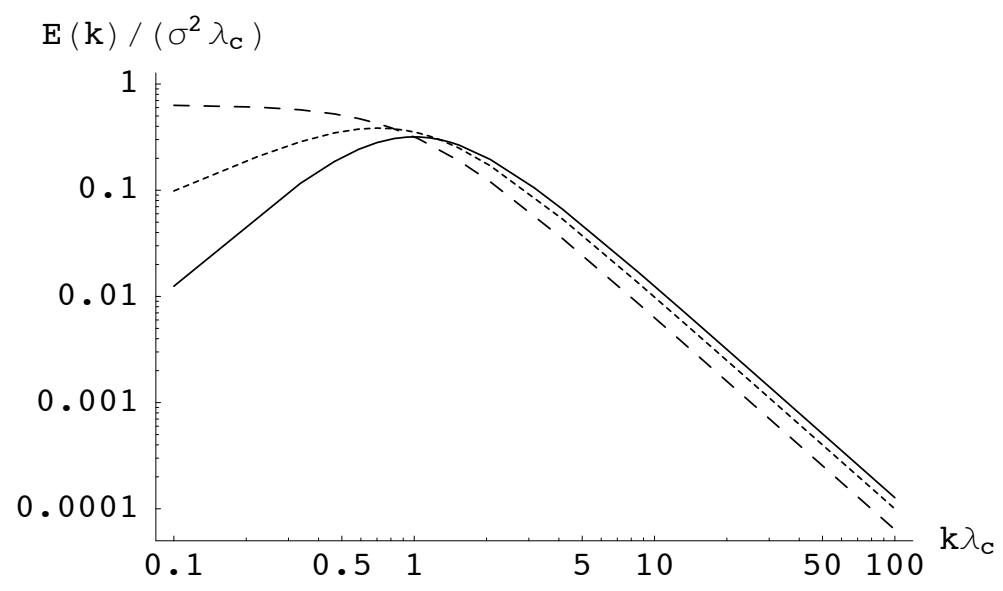

Figure 3.2: Comparison of normalized energy spectra $E(k) /\left(\sigma^{2} \lambda_{c}\right)$ in one dimension (dashed line), two dimensions (dotted line), and three dimensions (black solid line). The energy spectrum $E(k)$ is normalized by mean square deviations $\sigma^{2}=\left\langle\alpha^{2}\right\rangle$ and the correlation length $\lambda_{c}$. 
be obtained as follows. In three dimensions, Eq. (3.38) shows that $E(k)$ is proportional to $k^{-2}$, for $\lambda_{c} k>2 \pi$. In two dimensions, Eq. (3.35) shows that $E(k)$ is also proportional to $k^{-2}$, for $\lambda_{c} k>2 \pi$. In one dimensions, Eq. (3.32) shows that $E(k)$ is again proportional to $k^{-2}$, for $\lambda_{c} k>2 \pi$. To summarize, in the large wave number range $\lambda_{c} k>2 \pi$, the energy spectrum of the exponential decay is proportional to $k^{-2}$, regardless of the spatial dimension.

Note that the wave number range $k \lambda_{c}>2 \pi$ is called the inertial range. Assuming that the fluctuations in the inertial range are independent of viscosity, one can use the dimensional analysis to obtain the Kolmogorov spectrum $E(k) \propto k^{-5 / 3}$ $[17,18]$.

\subsection{Autopower spectrum}

Since $\Lambda(k)$ and $C(k, \tau=0)$ have been found, we may now use our formulae to calcualte the autopower spectra of the fluctuations.

We first consider the one-dimensional fluctuations. Inserting Eq. (3.31) into Eq. (3.4), we have

$$
S_{+}(k, \omega)=\left\langle\alpha^{2}\right\rangle \frac{2 \lambda_{c}}{1+k^{2} \lambda_{c}^{2}} \frac{1}{D k^{2}+i \omega} .
$$

We use the random walk formula for the effective diffusion coefficient

$$
D=\frac{\lambda_{c}^{2}}{\tau_{d}}=f_{d} \lambda_{c}^{2}
$$

where the correlation length $\lambda_{c}$ is the average step size of a random walk, and the diffusion time $\tau_{d}=1 / f_{d}$ is the average time between the steps of a random walk. With $S_{+}(k, \omega)$ given by Eq. (3.39), the contour integration of Eq. (3.19) gives

$$
S(r=0, \omega)=\left\langle\alpha^{2}\right\rangle \sqrt{2}\left[\frac{\sqrt{f_{d}} \sqrt{|\omega|}}{\omega^{2}+f_{d}^{2}}-\frac{\sqrt{2} f_{d}}{\omega^{2}+f_{d}^{2}}+\frac{f_{d}^{3 / 2}}{\sqrt{|\omega|}\left(\omega^{2}+f_{d}^{2}\right)}\right]
$$


or

$$
S(\omega)=\frac{\left\langle\alpha^{2}\right\rangle}{f_{d}} \frac{\sqrt{2}}{\nu^{2}+1}\left(\sqrt{|\nu|}-\sqrt{2}+\frac{1}{\sqrt{|\nu|}}\right),
$$

where $\nu=\omega / f_{d}$. The 1-d spectrum Eq. (3.42) is plotted in Fig. 3.3.

We next consider the two-dimensional fluctuations. Inserting Eq. (3.35) into Eq. (3.8), we have

$$
S(r=0, \omega)=\left\langle\alpha^{2}\right\rangle \int_{0}^{\infty} d k \frac{k \lambda_{c}^{2}}{\left(1+k^{2} \lambda_{c}^{2}\right)^{3 / 2}} \frac{2 D k^{2}}{D^{2} k^{4}+\omega^{2}} .
$$

This integral is convergent at $k \rightarrow \infty$, but the integrand at zero frequency is proportional to $k^{-1}$ for $k \rightarrow 0$. Thus we expect the spectrum to be divergent at $\omega=0$. If we let $D=f_{d} \lambda_{c}^{2}, \nu=\omega / f_{d}$ and $q=k \lambda_{c}$, then $D k^{2}=q^{2} f_{d}$ and Eq. (3.43) becomes

$$
S(\omega)=\frac{\left\langle\alpha^{2}\right\rangle}{f_{d}} \int_{0}^{\infty} d q \frac{q}{\left(1+q^{2}\right)^{3 / 2}} \frac{2 q^{2}}{q^{4}+\nu^{2}}
$$

The 2-d spectrum Eq. (3.44) is plotted in Fig. 3.3.

Finally, we consider the three-dimensional fluctuations. Inserting Eq. (3.38) into Eq. (3.8), we have

$$
S(r=0, \omega)=\left\langle\alpha^{2}\right\rangle \int_{0}^{\infty} d k \frac{4}{\pi} \frac{k^{2} \lambda_{c}^{3}}{\left(1+k^{2} \lambda_{c}^{2}\right)^{2}} \frac{2 D k^{2}}{D^{2} k^{4}+\omega^{2}}
$$

If we let $D=f_{d} \lambda_{c}^{2}, \nu=\omega / f_{d}$, and $q=k \lambda_{c}$, then $D k^{2}=q^{2} f_{d}$ and Eq. (3.45) becomes

$$
S(\omega)=\frac{\left\langle\alpha^{2}\right\rangle}{f_{d}} \frac{4}{\pi} \int_{0}^{\infty} d q \frac{q^{2}}{\left(1+q^{2}\right)^{2}} \frac{2 q^{2}}{q^{4}+\nu^{2}}
$$

The 3-d spectrum Eq. (3.46) is plotted in in Fig. 3.3.

To summarize, as shown in Fig. 3.3, when zero wave number is included in the $k$ integrals, the resulting autopower spectra decay monotonically and the resulting 1-d and 2-d autopower spectra are divergent at zero frequency. 


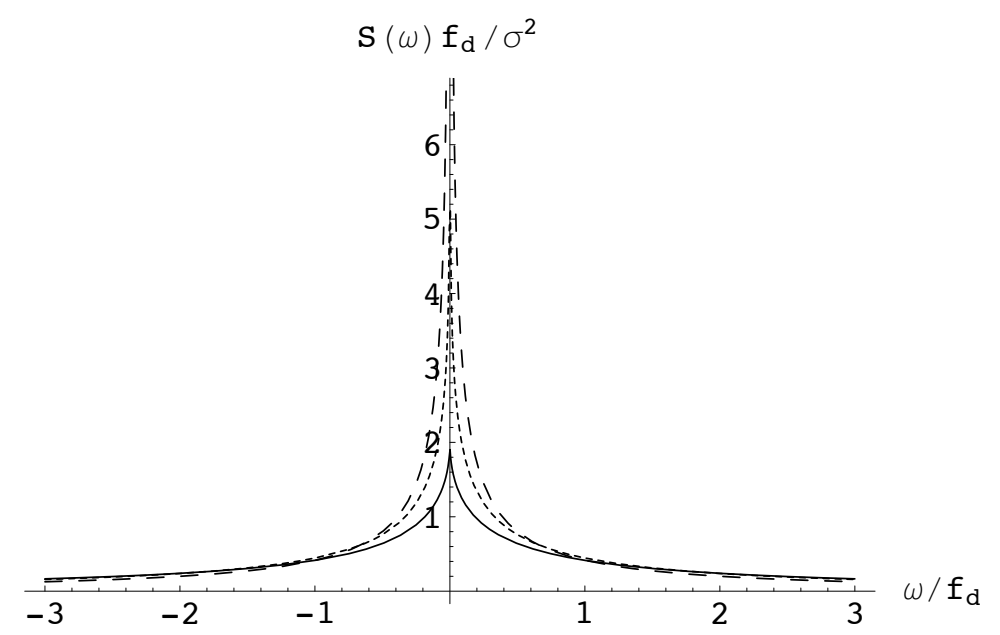

Figure 3.3: Comparison of the normalized autopower spectra $S(\omega) f_{d} / \sigma^{2}$ in one dimension (dashed line), two dimensions (dotted line), and three dimensions (black solid line). The autopower spectrum $S(\omega)$ is normalized by mean square deviations $\sigma^{2}=\left\langle\alpha^{2}\right\rangle$ and the diffusion frequency $f_{d}$.

Since the fluctuation wavelengths of interest are not expected to be infinite, we introduce a cutoff for the lower limit of the $k$ integrals to avoid the divergence.

On the other hand, for inhomogeneous systems that have gradients in the equilibrium profiles, we exclude very long wavelengths, so that the resulting smallscale fluctuations have homogeneous equilibrium background. The exact cutoff should be given by the diagnostics of the experiments. The correlation length is often shorter than the length scales of the equilibrium profile gradients. Thus the cutoff wave number is approximated by the minimum wave number of the inertial range:

$$
k_{c}=\frac{2 \pi}{\lambda_{c}},
$$

where $\lambda_{c}$ is the correlation length. Since the longest wavelength of the inertial range corresponds to the length scale at which the fluctuation energy is introduced, our approximation implies that the mean square value of small-scale fluctuations is de- 
termined by the wavelengths that are shorter than the correlation length. Therefore, Eq. (2.58) is approximated by

$$
\left\langle\alpha^{2}\right\rangle=\int_{k_{c}}^{\infty} d k E(k)
$$

and Eq. (3.8) is approximated by

$$
S(r=0, \omega)=\int_{k_{c}}^{\infty} d k E(k) \frac{2 D k^{2}}{D^{2} k^{4}+\omega^{2}}
$$

In view of Eqs. (3.48) and (3.6), we confirm that Eq. (3.49) satisfies the condition Eq. (2.11).

Recall that in the large wave number range $k>k_{c}$, the energy spectrum of the exponential decay

$$
E(k) \propto k^{-2}
$$

The proportionality constant can be obtained from the condition Eq. (3.48). Thus under the assumption of the fluctuation wave numbers $k>k_{c}$, regardless of the spatial dimension,

$$
E(k)=\left\langle\alpha^{2}\right\rangle \frac{k_{c}}{k^{2}}
$$

Eq. (3.51) shows that the amplitude of the energy spectrum is determined by the mean square deviations $\left\langle\alpha^{2}\right\rangle$ and the correlation length $\lambda_{c}$. The continuous energy spectrum indicates that many length scales are involved in random fluctuations.

Inserting Eq. (3.51) into Eq. (3.49), we have

$$
S(r=0, \omega) /\left\langle\alpha^{2}\right\rangle=\int_{k_{c}}^{\infty} d k \frac{k_{c}}{k^{2}} \frac{2 D k^{2}}{D^{2} k^{4}+\omega^{2}}=2 \int_{k_{c}}^{\infty} d k \frac{D k_{c}}{D^{2} k^{4}+\omega^{2}}
$$

Eq. (3.52) shows that many large wave numbers, i.e., short wavelengths contribute to the fluctuation power $S(r=0, \omega) d \omega$ in the frequency interval $d \omega$.

To calculate the integral, we write the effective diffusion coefficient of random 
fluctuations in the random walk form

$$
D=\frac{\omega_{d}}{k_{c}^{2}}=\frac{\lambda_{c}^{2}}{2 \pi \tau_{d}}=\frac{f_{d} \lambda_{c}^{2}}{2 \pi}
$$

where the diffusion frequency $\omega_{d}=2 \pi / \tau_{d}=2 \pi f_{d}$ is the step frequency of a random walk, and $\lambda_{c}=2 \pi / k_{c}$ is the step size of a random walk. If we let $\nu=\omega / \omega_{d}=f / f_{d}$ and $q=k / k_{c}$, then $D k^{2}=q^{2} \omega_{d}$ and Eq. (3.52) becomes

$$
S(\nu) /\left\langle\alpha^{2}\right\rangle=\frac{2}{\omega_{d}} \int_{1}^{\infty} \frac{d q}{q^{4}+\nu^{2}}=\frac{2}{3 \omega_{d}} H\left(-\nu^{2}\right),
$$

where $H\left(-\nu^{2}\right)$ represents the hypergeometric function ${ }_{2} F_{1}\left(\frac{3}{4}, 1 ; \frac{7}{4} ;-\nu^{2}\right)$. Note that the curvature of the function, i.e., the second derivative of the function, changes sign from negative to positive at the inflexional point $\nu=0.78$. For $\nu<0.2$, $H\left(-\nu^{2}\right) \approx 1-\frac{3}{7} \nu^{2}$. For $\nu>2, H\left(-\nu^{2}\right) \approx \nu^{-1.1}$.

Inserting $\nu=\omega / \omega_{d}$ into Eq. (3.54), we find that regardless of the spatial dimension, the autopower spectrum

$$
S(r=0, \omega)=\left\langle\alpha^{2}\right\rangle \frac{2}{3 \omega_{d}} H\left(-\omega^{2} / \omega_{d}^{2}\right) .
$$

This result is called the diffusion spectrum. Note that the lower limit of the $k$ integral, $k_{c}$, does not enter the final results. From Eq. (3.55) we see that the amplitude of the autopower spectrum is determined by the mean square deviations $\left\langle\alpha^{2}\right\rangle$ and the diffusion frequency $\omega_{d}$. Since $H(\omega=0)=1$, the spectrum has a finite maximum at zero frequency

$$
S_{\max }=S(\omega=0)=\left\langle\alpha^{2}\right\rangle \frac{2}{3 \omega_{d}} .
$$

As shown in Fig. 3.4, the frequency spectrum Eq. (3.55) is broad and continuous, 
which indicates that many time scales are involved in random fluctuations. Since

$$
\frac{2}{3 \omega_{d}} \int_{-\infty}^{\infty} \frac{d \omega}{2 \pi} H\left(-\omega^{2} / \omega_{d}^{2}\right)=1
$$

we confirm that the diffusion spectrum Eq. (3.55) satisfies the condition Eq. (2.11).

It is worth to parametrize the integral Eq. (3.52) in another way. If we let

$$
y=k \sqrt{\frac{D}{\omega}}=\frac{k}{k_{c}} \sqrt{\frac{\omega_{d}}{\omega}},
$$

then Eq. (3.52) becomes

$$
S(r=0, \omega) /\left\langle\alpha^{2}\right\rangle=2 \frac{\sqrt{\omega_{d}}}{\omega^{3 / 2}} \int_{y_{c}}^{\infty} \frac{d y}{y^{4}+1},
$$

where

$$
y_{c}=k_{c} \sqrt{\frac{D}{\omega}}=\sqrt{\frac{\omega_{d}}{\omega}} .
$$

Using the integration result

$$
\int_{y_{c}}^{y_{\max }} \frac{d y}{y^{4}+1}=\frac{H\left(-1 / y_{c}^{4}\right)}{3 y_{c}^{3}}-\frac{H\left(-1 / y_{\max }^{4}\right)}{3 y_{\max }^{3}}
$$

we again obtain Eq. (3.55) for the autopower spectrum. From Eq. (3.61) we see that even if the maximum wave number $k_{\max }$ of the measurement is finite, for $k_{\max }^{3}>>k_{c}^{3}$, the result Eq. (3.55) is still a good approximation.

\subsection{Autocorrelation function}

The autocorrelation function quantifies the correlations between different times at one spatial point. The inverse Fourier transform of the autopower spectrum gives 


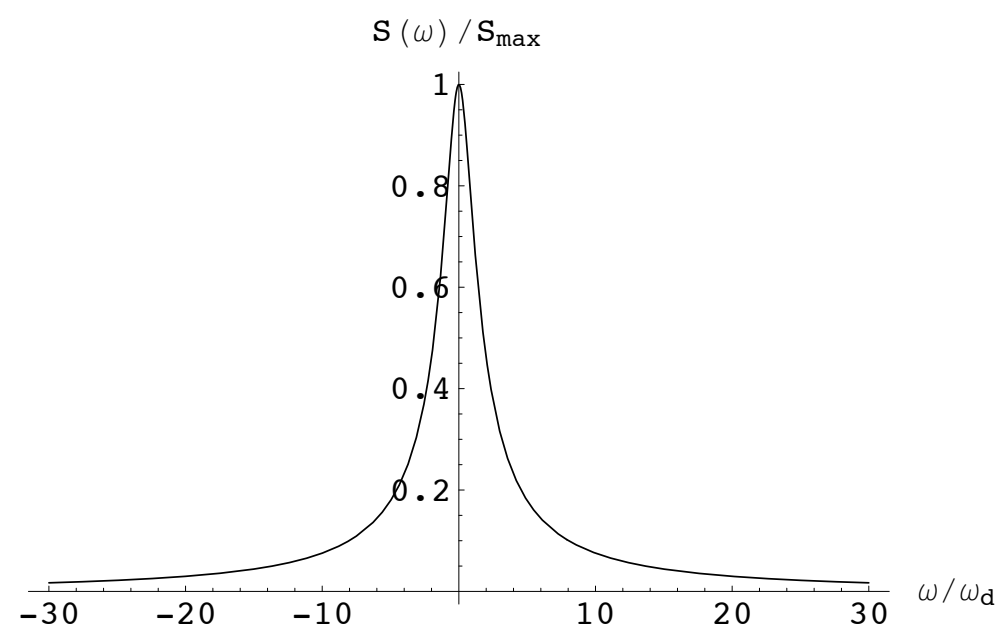

Figure 3.4: Normalized autopower spectrum $S(\omega) / S_{\max }$ versus normalized frequency $\omega / \omega_{d}$. The spectrum is normalized by its maximum value, $S_{\max }$, and the frequency is normalized by the diffusion frequency $\omega_{d}$.

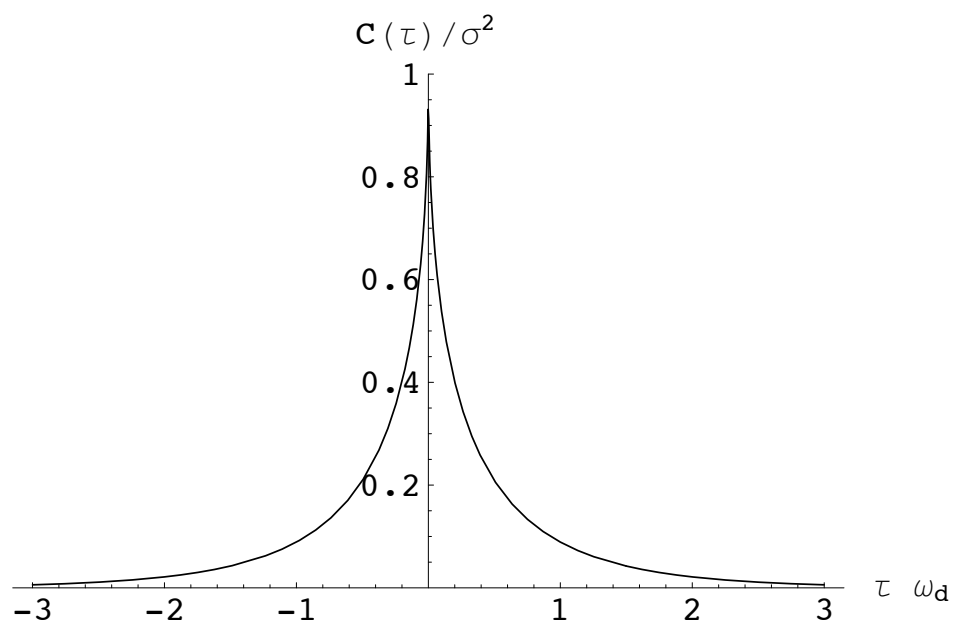

Figure 3.5: Normalized autocorrelation function $C(\tau) / \sigma^{2}$ versus normalized time difference $\tau \omega_{d}$. The autocorrelation function $C(\tau)$ is normalized by the mean square deviations $\sigma^{2}=\left\langle\alpha^{2}\right\rangle$, and the time differences $\tau$ are normalized by the diffusion frequency $\omega_{d}$. 
the autocorrelation function. Inserting Eq. (3.55) into Eq. (2.18), we have

$$
C(r=0, \tau)=\left\langle\alpha^{2}\right\rangle \int_{0}^{\infty} \frac{d \omega}{\pi} \cos (\omega \tau) \frac{2}{3 \omega_{d}} H\left(-\omega^{2} / \omega_{d}^{2}\right)
$$

If we let $T=\tau \omega_{d}$ and $\nu=\omega / \omega_{d}$, then $\omega \tau=\nu T$ and Eq. (3.62) becomes

$$
C(T) /\left\langle\alpha^{2}\right\rangle=\frac{2}{3} \int_{0}^{\infty} \frac{d \nu}{\pi} \cos (\nu T) H\left(-\nu^{2}\right)=e^{-|T|}-\sqrt{\pi|T|} \operatorname{erfc}(\sqrt{|T|})
$$

where the complementary error function $\operatorname{erfc}(z)=1-\operatorname{erf}(z)$ and $\operatorname{erf}(z)$ is the error function. Note that Eq. (3.63) decays faster than the exponential decay $e^{-|T|}$.

Inserting $T=\tau \omega_{d}$ into Eq. (3.63), we have

$$
C(r=0, \tau) /\left\langle\alpha^{2}\right\rangle=e^{-|\tau| \omega_{d}}-\sqrt{\pi|\tau| \omega_{d}} \operatorname{erfc}\left(\sqrt{|\tau| \omega_{d}}\right)
$$

This result is called the diffusion decay. As shown in Fig. 3.5, the slope of the diffusion decay Eq. (3.64) at $\tau=0$ is infinite and discontinuous. The slope discontinuity at the origin reflects inability of the Markovian approximation to resolve fine structure at time scales that are much shorter than the correlation time [12].

The correlation time, also known as the integral time scale, is defined by

$$
\tau_{c}=\int_{0}^{\infty} d \tau C(r=0, \tau) /\left\langle\alpha^{2}\right\rangle
$$

That is, the area under the autocorrelation function is equal to the mean square deviations $\left\langle\alpha^{2}\right\rangle$ times the correlation time $\tau_{c}$. Then it follows from the WienerKhintchin theorem Eq. (2.19) that

$$
S(r=0, \omega=0)=2 \int_{0}^{\infty} d \tau C(r=0, \tau)=2 \tau_{c}\left\langle\alpha^{2}\right\rangle
$$


The correlation time of the diffusion decay Eq. (3.64) is given by

$$
\tau_{c}=\int_{0}^{\infty} d \tau\left[e^{-\tau \omega_{d}}-\sqrt{\pi \tau \omega_{d}} \operatorname{erfc}\left(\sqrt{\tau \omega_{d}}\right)\right]=\frac{1}{3 \omega_{d}}
$$

We may use the reciprocal of the correlation time $1 / \tau_{c}$ to define the decay rate of the autocorrelation function at the height $C\left(\tau_{c}\right)=0.29\left\langle\alpha^{2}\right\rangle$. Since the power spectrum is the Fourier transform of the correlation function, the reciprocal of the correlation time indicates the width of the autopower spectrum $\Delta \omega=1 / \tau_{c}=3 \omega_{d}$ at the height $S(\Delta \omega)=0.315 S_{\max }$. Thus shorter correlation time, wider frequency spectrum.

Since $\omega_{d}=2 \pi / \tau_{d}=2 \pi f_{d}$, we have

$$
\tau_{c}=\frac{1}{3 \omega_{d}}=\frac{\tau_{d}}{6 \pi}=\frac{1}{6 \pi f_{d}}
$$

Thus the diffusion time $\tau_{d}$ is a measure of the correlation time, and the diffusion frequency $f_{d}$ is a measure of the decay rate, i.e., the spectrum width. We next rewrite the diffusion spectrum Eq. (3.55) as

$$
S(r=0, \omega)=2 \tau_{c}\left\langle\alpha^{2}\right\rangle H\left(-\omega^{2} / \omega_{d}^{2}\right)
$$

Since $H(\omega=0)=1$, we confirm that the diffusion spectrum Eq. (3.69) satisfies the condition Eq. (3.66). Finally, we rewrite the diffusion coefficient Eq. (3.53) as

$$
D=\frac{f_{d} \lambda_{c}^{2}}{2 \pi}=\frac{\lambda_{c}^{2}}{12 \pi^{2} \tau_{c}}
$$

Eq. (3.70) shows that the transport coefficients of random fluctuations are determined by the correlation length and the correlation time. 


\subsection{Oscillating decay}

The plasma turbulence experiments observe that often the autocorrelation function will not only decay but also oscillate at a nearly constant frequency. The regular oscillations suggest that there is a dominant frequency in the temporal correlation function. Note that the oscillation is not driven externally, for the amplitude of the oscillation decreases following the decaying envelope.

Hence we model the oscillation and decay of the autocorrelation function by a cosine function times a decaying function. That is, we write

$$
C(r=0, \tau)=\cos \left(\omega_{0} \tau\right) C_{d}(\tau)
$$

where $\omega_{0}=2 \pi f_{0}$ is a constant, $f_{0}$ is the oscillation frequency, and $C_{d}(\tau)$ represents the decaying function.

Consider the diffusion decay Eq. (3.64) for $C_{d}(\tau)$. Then Eq. (3.71) gives

$$
C(r=0, \tau) /\left\langle\alpha^{2}\right\rangle=\cos \left(\frac{\omega_{0}}{\omega_{d}} \tau \omega_{d}\right)\left[e^{-|\tau| \omega_{d}}-\sqrt{\pi|\tau| \omega_{d}} \operatorname{erfc}\left(\sqrt{|\tau| \omega_{d}}\right)\right]
$$

or

$$
C(r=0, \tau) /\left\langle\alpha^{2}\right\rangle=\cos \left(2 \pi f_{0} \tau\right)\left[e^{-|\tau| 2 \pi f_{d}}-\sqrt{\pi|\tau| 2 \pi f_{d}} \operatorname{erfc}\left(\sqrt{|\tau| 2 \pi f_{d}}\right)\right] .
$$

The oscillating decay Eq. (3.72) is compared to the diffusion decay Eq. (3.64) in

Fig. 3.6. Recall that the damped harmonic oscillator is also featured by oscillating decay. This analogy suggests that the regular oscillations in the temporal correlation function are related to the natural frequency of the fluctuations and the diffusion gives rise to the damping of the correlations. The quality factor of fluctuations may be defined by

$$
Q=\frac{\omega_{0}}{\omega_{d}}=\frac{f_{0}}{f_{d}}
$$




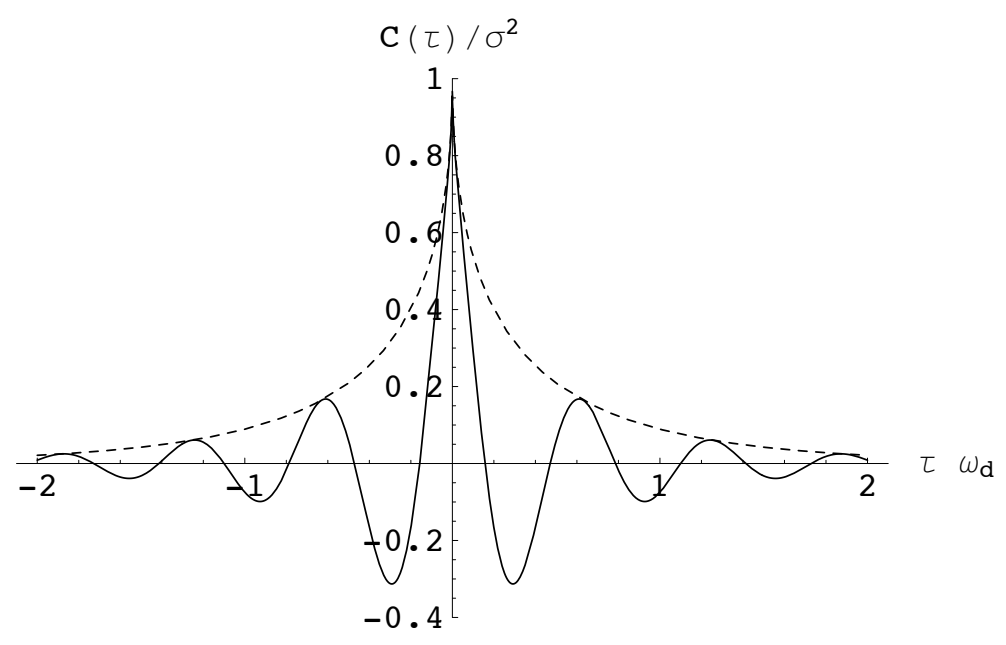

Figure 3.6: Comparison of the diffusion decay (dashed line) and the oscillating decay (solid line) for the case $\omega_{0} / \omega_{d}=10$. The autocorrelation function $C(\tau)$ is normalized by mean square deviations $\sigma^{2}=\left\langle\alpha^{2}\right\rangle$, and the time differences $\tau$ are normalized by the diffusion frequency $\omega_{d}$.

When the oscillation frequency $f_{0}$ is much larger than the decay rate $f_{d}$, the correlations are weakly damped with a large $Q$. The decay of the correlations is either oscillatory or not, depending on the amount of diffusion damping.

We next calculate the autopower spectrum. Inserting Eq. (3.71) into the Wiener-Khintchin theorem Eq. (2.19), we have

$$
S(r=0, \omega)=\frac{1}{2} \int_{-\infty}^{\infty} d \tau\left[e^{-i\left(\omega+\omega_{0}\right) \tau}+e^{-i\left(\omega-\omega_{0}\right) \tau}\right] C_{d}(\tau)
$$

or

$$
S(r=0, \omega)=\frac{1}{2}\left[S_{d}\left(\omega+\omega_{0}\right)+S_{d}\left(\omega-\omega_{0}\right)\right],
$$

where $S_{d}(\omega)$ is the temporal Fourier transform of $C_{d}(\tau)$.

Consider the diffusion spectrum Eq. (3.55) for $S_{d}(\omega)$. Then (3.75) gives

$$
S(r=0,|\omega|) /\left\langle\alpha^{2}\right\rangle=\frac{1}{2}\left(\frac{2}{3 \omega_{d}}\right)\left\{H\left[-\left(\omega+\omega_{0}\right)^{2} / \omega_{d}^{2}\right]+H\left[-\left(\omega-\omega_{0}\right)^{2} / \omega_{d}^{2}\right]\right\} .
$$




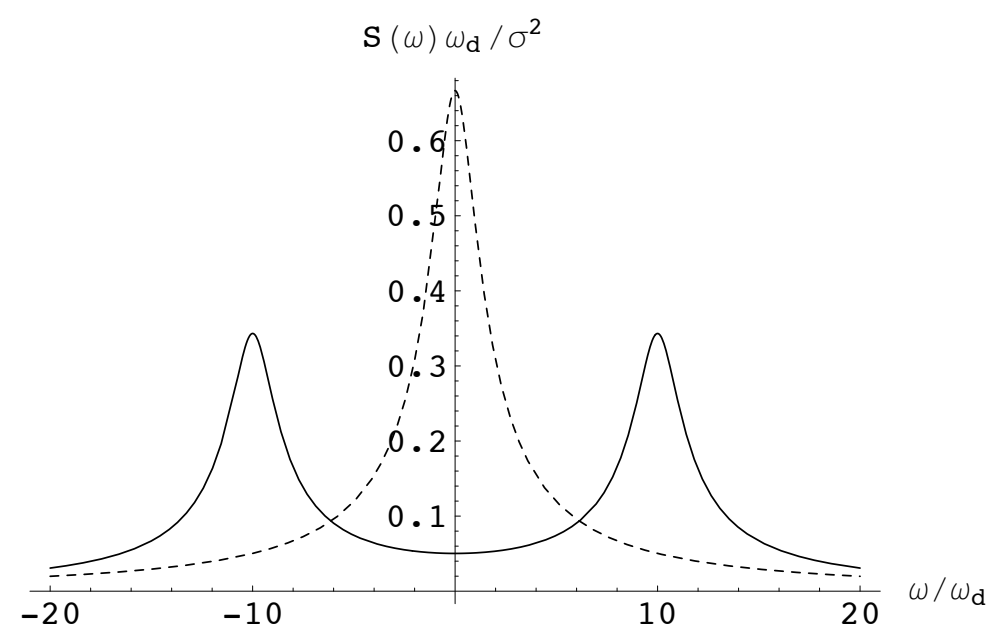

Figure 3.7: Comparison of the diffusion spectrum (dashed line) and the shifted spectrum (solid line) for the case $\omega_{0} / \omega_{d}=10$. The autopower spectrum $S(\omega)$ is normalized by mean square deviations $\sigma^{2}=\left\langle\alpha^{2}\right\rangle$ and the diffusion frequency $\omega_{d}$.

Thus the peak of the spectrum is shifted from zero frequency to two frequencies: $\omega_{0}$ and $-\omega_{0}$, and the amplitude of the resulting two peaks is reduced to be one half of the original peak. Since $\omega_{0}$ indicates the location of the spectrum peak, $\omega_{0}$ is also called the peak frequency of the spectrum. The shifted spectrum Eq. (3.76) is compared to the diffusion spectrum Eq. (3.55) in Fig. 3.7.

Since the autopower spectrum is even in $\omega$, it follows from Eq. (2.11) that

$$
\left\langle\alpha^{2}\right\rangle=\int_{0}^{\infty} d f 2 S(f)=\int_{0}^{\infty} d f G(f)
$$

where $G(f)=2 S(f)$ is the frequency spectrum usually measured in the experiments. Note that the spectral density $G(f)$ is defined to only have positive frequencies. It is clear that the autopower spectrum has units of power per frequency, here the power refers to the units of $\left\langle\alpha^{2}\right\rangle$. Using Eq. (3.76) we have

$$
G(f)=2 S(f)=\frac{\left\langle\alpha^{2}\right\rangle}{3 \pi f_{d}}\left\{H\left[-\left(f+f_{0}\right)^{2} / f_{d}^{2}\right]+H\left[-\left(f-f_{0}\right)^{2} / f_{d}^{2}\right]\right\}
$$




\section{Chapter 4}

\section{Convection-Diffusion Model}

The peak of the fluctuation spectrum has been studied by various experiments and theories [16, 19, 20, 21, 22, 23]. In this chapter, we will use the convection-diffusion model to explain the physical meaning of the peak frequency of the fluctuation spectrum.

\subsection{Convection-diffusion equation}

For a wide variety of physical situations, the relaxation of fluctuations involves a combination of fluid motion and diffusion. Hence the evolution of the conditional mean is modeled by the convection-diffusion equation

$$
\frac{\partial}{\partial t}\langle\alpha(\boldsymbol{x}, t)\rangle_{0}+\boldsymbol{U} \cdot \nabla\langle\alpha(\boldsymbol{x}, t)\rangle_{0}-D \nabla^{2}\langle\alpha(\boldsymbol{x}, t)\rangle_{0}=0
$$

where $\boldsymbol{U}$ is the effective flow velocity and $D$ is the effective diffusion coefficient.

Comparing Eq. (2.2) with Eq. (4.1), we obtain the convection-diffusion response function

$$
\Lambda\left(\boldsymbol{x}-\boldsymbol{x}^{\prime}\right)=-D \nabla^{2} \delta\left(\boldsymbol{x}-\boldsymbol{x}^{\prime}\right)-\boldsymbol{U} \cdot \boldsymbol{\nabla} \delta\left(\boldsymbol{x}-\boldsymbol{x}^{\prime}\right)
$$


or

$$
\Lambda(\boldsymbol{r})=-D \nabla^{2} \delta(\boldsymbol{r})-\boldsymbol{U} \cdot \nabla \delta(\boldsymbol{r})
$$

The Fourier transform of $\Lambda(\boldsymbol{r})$ gives

$$
\Lambda(\boldsymbol{k})=D k^{2}-i \boldsymbol{k} \cdot \boldsymbol{U}
$$

Thus in the convection-diffusion model, $\Lambda(\boldsymbol{k})$ is complex. Note that the real part of $\Lambda(\boldsymbol{k})$ represents isotropy, and the imaginary part of $\Lambda(\boldsymbol{k})$ represents anisotropy. Then the spectrum formula Eq. (2.76) gives

$$
S(\boldsymbol{k}, \omega)=C(\boldsymbol{k}, \tau=0) \frac{2 D k^{2}}{D^{2} k^{4}+(\omega-\boldsymbol{k} \cdot \boldsymbol{U})^{2}}
$$

\subsection{Anisotropy in magnetized plasmas}

Because of the strong magnetic fields, the relaxation of fluctuations in the magnetized plasma is anisotropic: the correlation structure in the direction parallel to the magnetic field is very different from the one in the two directions perpendicular to the magnetic field. The parallel correlation length $\lambda_{c \|}$ is much longer than the perpendicular correlation length $\lambda_{c \perp}$. Thus we expect that fluid motions dominate along the magnetic field, but diffusion processes dominate in the perpendicular directions. Then the convection-diffusion equation becomes

$$
\frac{\partial}{\partial t}\langle\alpha(\boldsymbol{x}, t)\rangle_{0}+U_{\|} \nabla_{\|}\langle\alpha(\boldsymbol{x}, t)\rangle_{0}-D_{\perp} \nabla_{\perp}^{2}\langle\alpha(\boldsymbol{x}, t)\rangle_{0}=0
$$

where $U_{\|}$is the effective flow velocity along the magnetic field, and $D_{\perp}$ is the effective diffusion coefficient perpendicular to the magnetic field. Then Eq. (4.3) becomes

$$
\Lambda(\boldsymbol{k})=D_{\perp} k_{\perp}^{2}-i k_{\|} U_{\|}
$$


and Eq. (4.4) becomes

$$
S(\boldsymbol{k}, \omega)=C(\boldsymbol{k}, \tau=0) \frac{2 D_{\perp} k_{\perp}^{2}}{D_{\perp}^{2} k_{\perp}^{4}+\left(\omega-k_{\|} U_{\|}\right)^{2}}
$$

We assume that the spatial correlations at one instant of time take the form

$$
\left\langle\alpha(\boldsymbol{x}, t) \alpha\left(\boldsymbol{x}^{\prime}, t\right)\right\rangle=C(\boldsymbol{r}, \tau=0)=\left\langle\alpha^{2}\right\rangle e^{-\left|\boldsymbol{r}_{\perp}\right| / \lambda_{c \perp}} \cos \left(k_{0} r_{\|}\right),
$$

where the constant $k_{0}$ indicates a dominant parallel wave number. Then the formula Eq. (2.78) gives

$$
S(\omega)=\int_{-\infty}^{\infty} \frac{d k_{\|}}{2 \pi} \frac{2 \pi}{2}\left[\delta\left(k_{\|}-k_{0}\right)+\delta\left(k_{\|}+k_{0}\right)\right] 2 \operatorname{Re} \int \frac{d^{2} k_{\perp}}{(2 \pi)^{2}} \frac{C\left(k_{\perp}, \tau=0\right)}{D_{\perp} k_{\perp}^{2}+i\left(\omega-k_{\|} U_{\|}\right)}
$$

The equilibrium plasma profiles are not uniform in the perpendicular directions, so we introduce the cutoff wave number for the perpendicular diffusion. Then Eq. (4.9) becomes

$$
S(r=0, \omega)=\int_{k_{c \perp}}^{\infty} d k_{\perp} E\left(k_{\perp}\right) \frac{1}{2}\left[\frac{2 D_{\perp} k_{\perp}^{2}}{D_{\perp}^{2} k_{\perp}^{4}+\left(\omega+\omega_{0}\right)^{2}}+\frac{2 D_{\perp} k_{\perp}^{2}}{D_{\perp}^{2} k_{\perp}^{4}+\left(\omega-\omega_{0}\right)^{2}}\right],
$$

where $\omega_{0}=k_{0} U_{\|}$is a constant, and $E\left(k_{\perp}\right)$ is given by Eq. (3.51):

$$
E\left(k_{\perp}\right)=\left\langle\alpha^{2}\right\rangle \frac{k_{c \perp}}{k_{\perp}^{2}}
$$

In view of Eqs. (3.49) and (3.55), we obtain the shifted autopower spectrum

$$
S(r=0,|\omega|)=\frac{1}{2}\left\{\frac{2\left\langle\alpha^{2}\right\rangle}{3 \omega_{d}} H\left[-\left(\omega+\omega_{0}\right)^{2} / \omega_{d}^{2}\right]+\frac{2\left\langle\alpha^{2}\right\rangle}{3 \omega_{d}} H\left[-\left(\omega-\omega_{0}\right)^{2} / \omega_{d}^{2}\right]\right\} .
$$

Note that this is exactly the same result as Eq. (3.76), which is obtained by assuming that there is a dominant frequency $\omega_{0}$ in the temporal correlation function. 


\section{Chapter 5}

\section{Comparison with Experiment}

In this chapter, we will use noise theory to study the temporal correlations of the plasma random fluctuations. We will compare the autocorrelation function and autopower spectral density calculated from noise theory with the observed plasma density fluctuations from tokamak and helimak experiments.

\subsection{Fluctuations in magnetized plasmas}

During a steady-state plasma discharge, the fluctuations in the magnetized plasma usually have the following properties $[15,24]$ : the time series of the signals are nearly random; in the two dimensions perpendicular to the strong magnetic field, the statistical properties of small-scale fluctuations are roughly homogeneous and isotropic, since the correlation length of the fluctuations is shorter than the length scales of the background plasma density and temperature gradients. Therefore, we may use Eqs. (3.73) and (3.78) to explain the shape and height of the measured autocorrelation function and autopower spectrum.

To compare the theory with experiments, we use the measured mean square deviations $\left\langle\alpha^{2}\right\rangle$ and the observed oscillation frequency, i.e., the peak frequency $f_{0}$. 
To fit the experimental date, we only choose the value of the decay rate, i.e., the spectrum width $f_{d}$. That is, the theory predicts the time dependence of the autocorrelation function and the frequency dependence of the autopower spectrum.

\subsection{Data processing}

In order to compare the discrete spectrum data with the continuous power spectral density, the raw data are processed as follows.

For tokamak experiments, the raw data represent the autopower of the fractional fluctuations $\alpha$, in the sense that the sum of raw data is equal to the mean square deviations $\sigma^{2}=\left\langle\alpha^{2}\right\rangle$, and the fluctuation level is equal to the standard devi-

ation $\sigma=\sqrt{\left\langle\alpha^{2}\right\rangle}$. The raw data are divided by the frequency bin $\Delta f=3.906 \mathrm{kHz}$, so that the processed data are equivalent to the spectral density $G(f)$, which has units of power per frequency, here the power refers to the units of $\left\langle\alpha^{2}\right\rangle$.

For helimak experiments, the spectrum data and the autocorrelation data are obtained independently, in the sense that the spectrum data are obtained from the ensemble averages of the Fast Fourier Transform of the time series and the autocorrelation data are obtained from the time averages of the same time series. The raw autocorrelation data are normalized by the mean square deviations $\sigma^{2}=$ $\left\langle\alpha^{2}\right\rangle$, so that $C(\tau) / \sigma^{2}$ is dimensionless. The raw Fourier spectra data are the square root of the autopower, in the sense that the sum of the square of the raw data is equal to the mean square deviations $\sigma^{2}=\left\langle\alpha^{2}\right\rangle$. The fluctuation level is given by the ratio of the standard deviation $\sigma$ to the mean value of the signal. The raw data are divided by the standard deviation $\sigma$ and the square root of the frequency bin $\Delta f=$ 13.7329 Hz, so that the processed data are equivalent to the normalized spectral density $\sqrt{G(f)} / \sigma$. Since the spectral density $G(f)$ is normalized by the mean square deviations $\sigma^{2}=\left\langle\alpha^{2}\right\rangle$, the quantity $G(f) / \sigma^{2}$ has units of inverse frequency. Note that the data in Fig. 3 of Ref. [25] were taken from argon discharge 508020092 at 


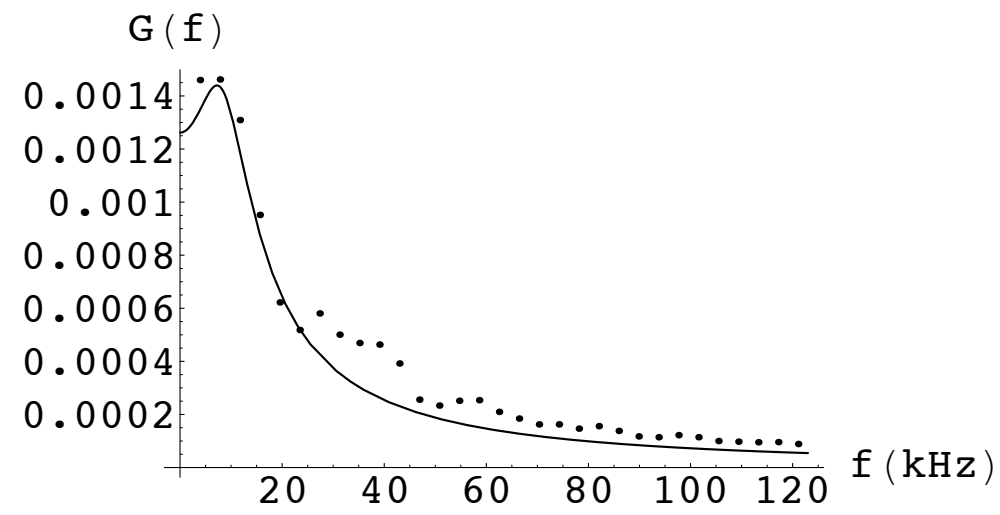

Figure 5.1: Power spectral density $G(f)$ (power $/ \mathrm{kHz}$ ) versus frequency $f(\mathrm{kHz})$. The dots represent the autopower spectrum data of the fractional plasma density fluctuations from tokamak experiments. The curve is given by Eq. (3.78).

the radial position $106.5 \mathrm{~cm}$, and the Fourier spectra data are divided by the mean value of the signal and $\sqrt{\Delta f}$, so that the resulting data represent the square root of spectral density $G(f)$ (power/Hz) for the fractional fluctuations.

\subsection{Tokamak experiments}

The tokamak is a toroidal plasma confinement device. The magnetic fields in the tokamak are the combination of a strong toroidal field and a weak poloidal field. The plasma density fluctuations in the edge of the Texas Experimental Tokamak have been measured [26]. The data in Fig. 5.1 were taken from deuterium discharge 230000 at minor radius $27.5 \mathrm{~cm}$, where the limiter was located. Note that Fig. 5.1 is reproduced from Fig. 1 of Ref. [25]. From the experimental data, one can obtain the peak frequency $f_{0} \approx 8 \mathrm{kHz}$ and the mean square deviations $\sigma^{2} \approx 0.055$. Thus the fluctuation level $\approx 24 \%$. To fit the experimental data, we use $f_{d} \approx 5.4 \mathrm{kHz}$ for the spectrum width. Using Eq. (3.74) we calculate the quality factor $Q=f_{0} / f_{d} \approx 1.5$. 
Using Eq. (3.68) we obtain the correlation time $\tau_{c}=1 /\left(6 \pi f_{d}\right) \approx 0.01 \mathrm{~ms}$. The perpendicular correlation length $\lambda_{c \perp}$ near the limiter was about $3 \mathrm{~cm}$ [15]. Using Eq. (3.70) we estimate the perpendicular diffusion coefficient $D_{\perp}=f_{d} \lambda_{c \perp}^{2} /(2 \pi) \approx$ $0.77 \mathrm{~m}^{2} / \mathrm{s}$.

\section{$5.4 \quad$ Helimak experiments}

The helimak is a toroidal device with a rectangular cross section. The magnetic fields in the helimak are the combination of a strong toroidal field and a weak vertical field. The distance along the field line from the bottom to the top of the machine is called the connection length. The helimak experiments [16] operate in a steady state for tens of seconds, giving excellenct statistics for turbulence. The plasma density fluctuations are measured by an array of probes in the radial direction of the vacuum vessel. The strongly peaked spectra are observed in the low magnetic field side, where the radial positions are larger than $100 \mathrm{~cm}$.

We first analyze the plasma density fluctuation data from argon discharge 508020092 with the connection length $21 \mathrm{~m}$.

The linear plot the fluctuation spectra is shown in Fig. 5.2. The autocorrelations are shown in Fig. 5.3. The time series data were taken at the radial position $114.5 \mathrm{~cm}$. From the experimental data, one can obtain the fluctuation level $\approx 33 \%$ and the peak frequency, i.e., the oscillation frequency $f_{0} \approx 1410 \mathrm{~Hz}$. To fit the experimental data, we use $f_{d} \approx 95 \mathrm{~Hz}$ for the spectrum width, i.e., the decay rate. Thus the quality factor $Q=f_{0} / f_{d} \approx 15$ and the correlation time $\tau_{c}=1 /\left(6 \pi f_{d}\right) \approx 0.56$ ms. For the perpendicular correlation length $\lambda_{c \perp} \approx 10 \mathrm{~cm}$, we estimate the local perpendicular diffusion coefficient $D_{\perp}=f_{d} \lambda_{c \perp}^{2} /(2 \pi) \approx 0.15 \mathrm{~m}^{2} / \mathrm{s}$.

In the same way, we can obtain the decay rate, i.e., the spectrum width $f_{d}$ of the plasma density fluctuations at different radial positions. The results are listed in Table 5.1. From Table 5.1, we see that as the radial positions move to 


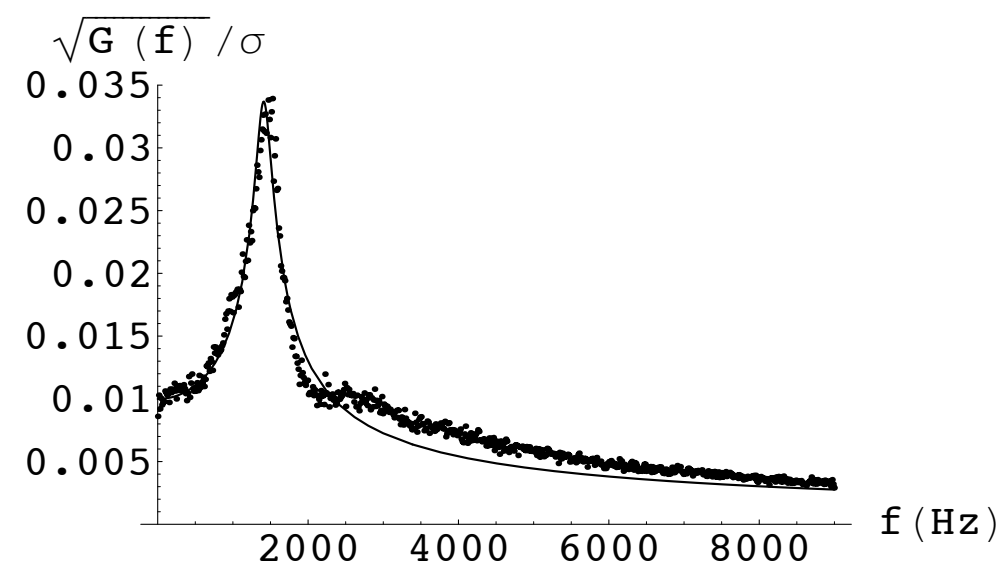

Figure 5.2: Square root of normalized spectral density $\sqrt{G(f)} / \sigma(1 / \sqrt{\mathrm{Hz}})$ versus frequency $f(\mathrm{~Hz})$. The dots represent the square root of the autopower spectrum data of the plasma density fluctuations from helimak experiments with short connection length at the radial position $114.5 \mathrm{~cm}$. The curve is given by the square root of Eq. (3.78).

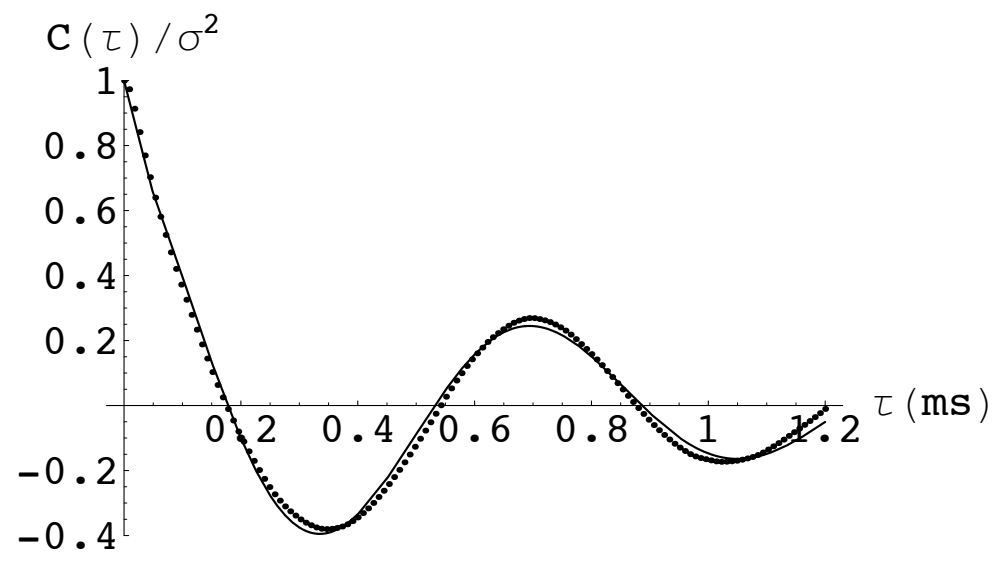

Figure 5.3: Normalized autocorrelation function $C(\tau) / \sigma^{2}$ versus time difference $\tau$ $(\mathrm{ms})$. The dots represent the autocorrelation data of the plasma density fluctuations from helimak experiments with short connection length at the radial position 114.5 $\mathrm{cm}$. The curve is given by Eq. (3.73). 
Table 5.1: The observed fluctuation level, the observed oscillation frequency, i.e., the peak frequency $f_{0}$, and the decay rate, i.e., the spectrum width $f_{d}$ of the plasma density fluctuations from helimak experiments with short connection length at different radial positions.

\begin{tabular}{cccc}
\hline \hline Radial position $(\mathrm{cm})$ & Fluctuation level & $f_{0}(\mathrm{~Hz})$ & $f_{d}(\mathrm{~Hz})$ \\
\hline 110.5 & 0.23 & 1410 & 90 \\
114.5 & 0.33 & 1410 & 95 \\
118.5 & 0.38 & 1410 & 110 \\
122.5 & 0.31 & 1410 & 160 \\
\hline \hline
\end{tabular}

the plasma edge, the decay rate, i.e., the spectrum width becomes larger, while the oscillation frequency, i.e., the peak frequency has little variation. Presumably the correlation length is constant in this local region. Thus the random walk formula $D_{\perp}=f_{d} \lambda_{c \perp}^{2} /(2 \pi)$ tells us that the observations of wider frequency spectra or faster decay of the autocorrelation functions imply that transport coefficients become larger. In fact, we expect that transport coefficients will increase towards the edge of the plasma [3].

It is remarkable that the results derived from noise theory are independent of the form of the probability distribution. The measured probability distributions at different radial positions are shown in Fig. 5.4. By definition, the average of the fluctuations $\langle\alpha\rangle=0$ and the mean square deviations

$$
\sigma^{2}=\left\langle\alpha^{2}\right\rangle=\int d \alpha P(\alpha) \alpha^{2}
$$

where $\sigma$ is the standard deviation of the signal. For the data in Fig. 5.4, the positive sigma events correspond to the lower plasma density events. Recall that the Gaussian distribution takes the form

$$
P(\alpha)=P_{\max } e^{-\alpha^{2} /\left(2 \sigma^{2}\right)}
$$




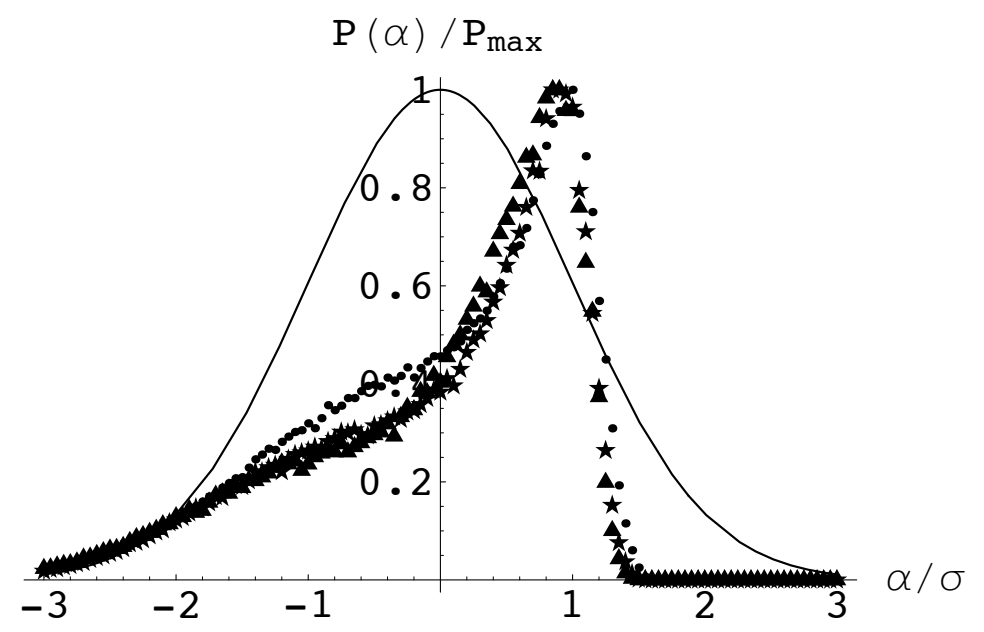

Figure 5.4: Normalized probability distribution $P(\alpha) / P_{\max }$ versus the normalized fluctuations $\alpha / \sigma$ at the radial positions: $110.5 \mathrm{~cm}$ (dots), $114.5 \mathrm{~cm}$ (stars), and $118.5 \mathrm{~cm}$ (triangles). The curve represents the Gaussian distribution Eq. (5.2). The probability is normalized by its maximum value, $P_{\max }$, and the fluctuations of the signal $\alpha$ are normalized by the standard deviation of the signal, $\sigma$.

where

$$
P_{\max }=\frac{1}{\sqrt{\pi 2 \sigma^{2}}}
$$

From Fig. 5.4, we see that the probability distribution of plasma density fluctuations is far from Gaussian. Using the measured probability distributions, one can test if the observed fluctuations are statistically homogeneous and isotropic. The data in Fig. 5.4 for different radial positions collapse onto a single curve when normalized. This immediately tells us that the plasma density fluctuations are statistically homogeneous, since the probability distributions are translationally invariant in this local region.

To investigate how the connection lengths of the magnetic field lines affect the plasma fluctuations, we now analyze the plasma density fluctuation data from argon discharge 508020095 with the connection length $74 \mathrm{~m}$.

The autocorrelations are shown in Fig. 5.6. The linear and log-log plots of 


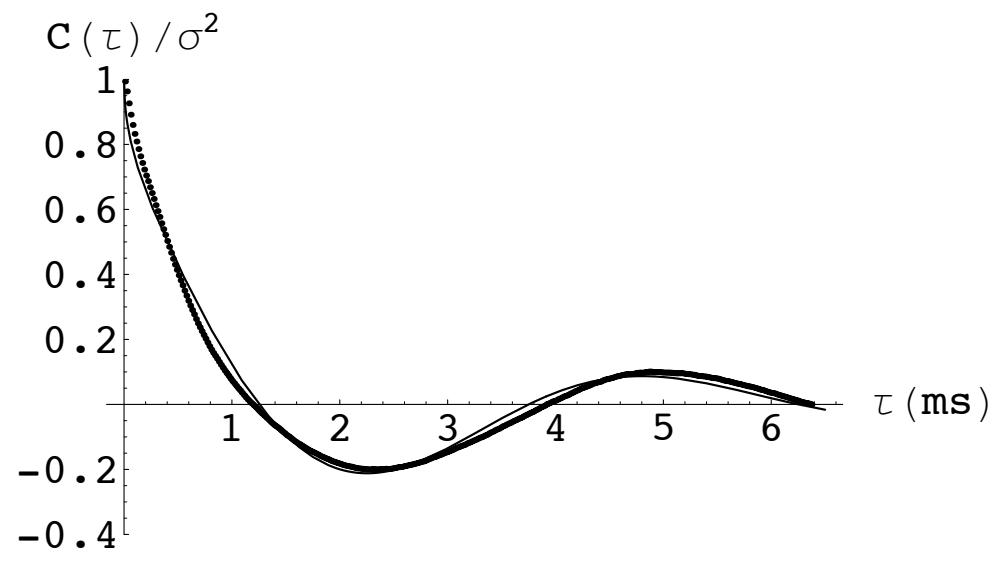

Figure 5.5: Normalized autocorrelation function $C(\tau) / \sigma^{2}$ versus time difference $\tau$ (ms). The dots represent the autocorrelation data of the plasma density fluctuations from helimak experiments with long connection length at the radial position 110.5 $\mathrm{cm}$. The curve is given by Eq. (3.73).

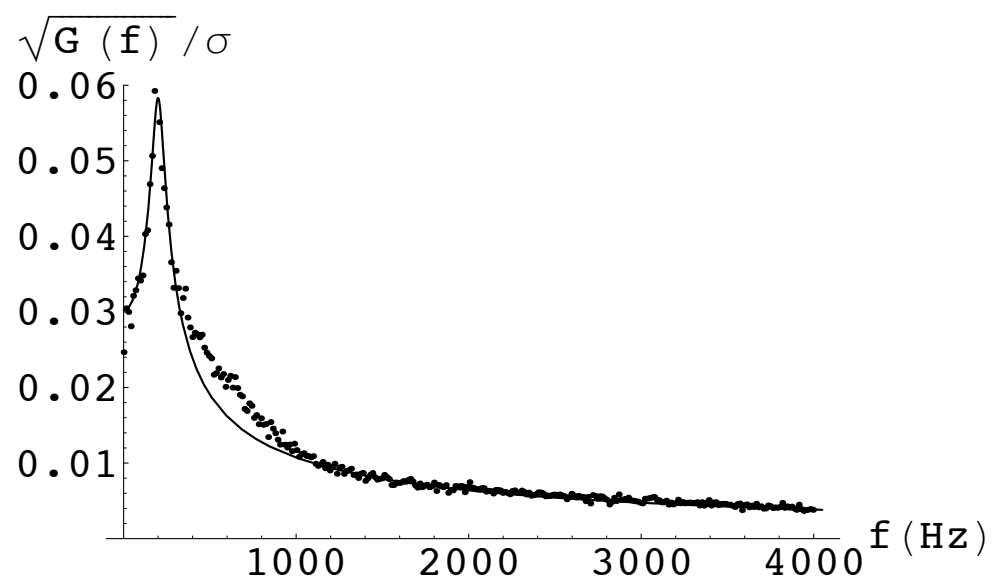

Figure 5.6: Square root of normalized spectral density $\sqrt{G(f)} / \sigma(1 / \sqrt{\mathrm{Hz}})$ versus frequency $f(\mathrm{~Hz})$. The dots represent the square root of the autopower spectrum data of the plasma density fluctuations from helimak experiments with long connection length at the radial position $110.5 \mathrm{~cm}$. The curve is given by the square root of Eq. (3.78). 


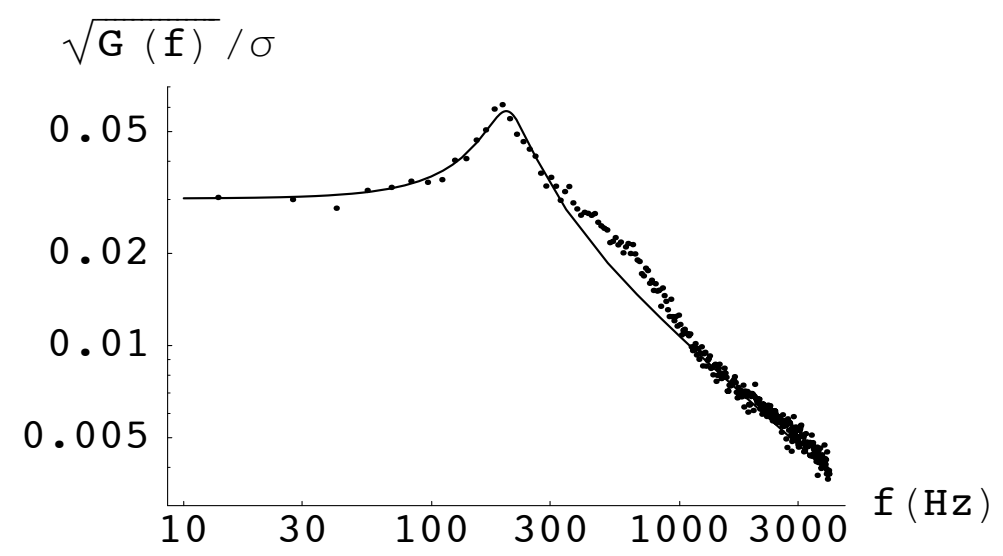

Figure 5.7: Square root of normalized spectral density $\sqrt{G(f)} / \sigma(1 / \sqrt{\mathrm{Hz}})$ versus frequency $f(\mathrm{~Hz})$. The dots represent the square root of the autopower spectrum data of the plasma density fluctuations from helimak experiments with long connection length at the radial position $110.5 \mathrm{~cm}$. The curve is given by the square root of Eq. (3.78).

the fluctuation spectra are shown in Figs. 5.6 and 5.7, respectively. The time series data were taken at the radial position $110.5 \mathrm{~cm}$. From the experimental data, one can obtain the fluctuation level $\approx 27 \%$ and the peak frequency, i.e., the oscillation frequency $f_{0} \approx 200 \mathrm{~Hz}$. To fit the experimental data, we use $f_{d} \approx 33 \mathrm{~Hz}$ for the spectrum width, i.e., the decay rate. Thus the quality factor $Q=f_{0} / f_{d} \approx 6$ and the correlation time $\tau_{c}=1 /\left(6 \pi f_{d}\right) \approx 1.6 \mathrm{~ms}$.

Comparing the temporal correlations for short and long connection lengths, we see that in the case of shorter connection length, the autopower spectrum has higher peak frequency and wider width; the corresponding autocorrelation function has faster oscillation and shorter correlation time. 


\subsection{Discussion}

In summary, the temporal correlations of random fluctuations have the following key features: the autocorrelation function has a fast decay in the small $\tau$ region and a slower decay in the large $\tau$ region. Since the autopower spectrum is equal to the Fourier transform of the autocorrelation function, the corresponding autopower spectrum has a long tail in the high frequency region and a narrow maximum near the peak frequency.

Note that the experimental data are more likely to be biased by the measurement noise, when the amplitude of the correlations is small. This may explain the disagreement between the data points and the theoretical curves at some low correlation regions. In addition, the disagreement may be caused by the fact that the statistics of the observed fluctuations are not perfectly homogeneous and isotropic.

Finally, we remark that the exponential decay and the Gaussian function cannot fit the full time range of the autocorrelation data and the corresponding Fourier transforms: the Lorentzian and the Gaussian cannot fit the full frequency range of the spectrum data. 


\section{Chapter 6}

\section{Summary and Conclusions}

We have used noise theory to study the time and space correlations of stationary Markovian fluctuations that are statistically homogeneous and isotropic.

In Chapter 1 we have derived the Wiener-Khintchin theorem and discussed the time-reversal symmetry. The relaxation of stationary Markov processes has been described by means of the conditional mean. Based on the Markovian relaxation, we have obtained the discrete-index formulae of the power spectra, which are the essential results of noise theory.

In Chapter 2 we have defined the following statistical quantities of homogeneous fluctuations: the correlation function, the power spectrum, the wavenumber spectrum, and the wavenumber-frequency spectrum. The relations between these statistical quantities have been derived. Based on the discrete-index formulae, we have obtained the general formulae of the wavenumber-frequency spectra, which are the essential tools of our theory. We have also defined the energy spectrum of isotropic fluctuations.

In Chapter 3 we have used the diffusion equation to model the Markovian relaxation of homogeneous isotropic fluctuations. The delta function for the spatial correlations has been used to demonstrate the general features of the time and space 
correlations due to the diffusion. We find that the diffusion process can give rise to the decay of the correlation function and a broad frequency spectrum of random fluctuations. For physical systems with finite correlation length, we have used the exponential decay to model the spatial correlations at one instant of time. It is found that the energy spectra of the exponential decay are proportional to $k^{-2}$ in the large wave number range. Assuming that the mean square value of smallscale fluctuations are determined by the wavelengths shorter than the correlation length, we have obtained the analytic results of the autopower spectrum and the autocorrelation function. The results are independent of spatial dimensions. We find that in the diffusion model the autopower spectrum has a single peak at zero frequency and the autocorrelation function purely decays away. We also find that the fluctuation-induced transport coefficients may be estimated by the correlation length and the correlation time. Assuming that there is a dominant frequency in the temporal correlations, the oscillation and decay of the autocorrelation function have been modeled by a cosine function times the decay due to diffusion. As a consequence, the maximum of the corresponding autopower spectrum is shifted from zero frequency to the dominant frequency.

In Chapter 4 we have used the convection-diffusion equation to describe the relaxation of fluctuations that involves fluid motion and diffusion. We have shown that the product of the wave number and the flow velocity can shift the peak of the spectra away zero frequency.

In Chapter 5 we have investigated the applications of noise theory to plasma fluctuations. The theory has been compared with plasma density fluctuations from tokamak and helimak experiments. The quantitative comparisons show that the theory can predict the shape of the autopower spectrum and the autocorrelation function of the observed plasma fluctuations over a wide range of frequencies and time scales. The theory can also relate the amplitude of the spectra to other spec- 
tral characteristics, such as correlation times. In particular, we have shown that the fluctuation-induced transport coefficients are proportional to the width of the fluctuation spectra.

Finally, we outline our future work. First, we would like to calculate the crosscorrelations, i.e., the temporal correlations between two spatial points. Second, we should investigate the physics of the correlation time and the peak frequency by a thorough comparison of these characteristic frequencies with the plasma collision and drift frequencies. Third, we need to investigate the role of $k_{\|} U_{\|}$and $\boldsymbol{k}_{\perp} \cdot \boldsymbol{U}_{\perp}$ in shifting the fluctuation spectra of the magnetized plasma. 


\section{Bibliography}

[1] R. D. Hazeltine and F. L. Waelbroeck, The Framework of Plasma Physics (Perseus Books, Reading, Massachusetts, 1998).

[2] R. D. Hazeltine and J. D. Meiss, Plasma Confinement (Dover Publications, Mineola, New York, 2003).

[3] K. W. Gentle, Rev. Mod. Phys. 67, 809 (1995).

[4] H. B. Callen and T. A. Welton, Phys. Rev. 83, 34 (1951).

[5] L. Onsager and S. Machlup, Phys. Rev. 91, 1505 (1953).

[6] M. Lax, Rev. Mod. Phys. 38, 541 (1966).

[7] E. C. Taylor and G. G. Comisar, Phys. Rev. 132, 2379 (1963).

[8] C. R. Oberman and E. A. Williams, in Basic Plasma Physics, edited by A. A. Gaeleev and R. N. Sudan (North-Holland, Amsterdam, 1983), vol. 1.

[9] R. H. Kraichnan, Phys. Rev. Lett. 72, 1016 (1994).

[10] J. A. Krommes, Phys. Rep. 360, 1 (2002).

[11] R. D. Hazeltine and S. M. Mahajan, Phys. Plasmas 11, 5430 (2004).

[12] M. Lax, Rev. Mod. Phys. 32, 25 (1960). 
[13] M. Lax and P. Mengert, J. Phys. Chem. Solids 14, 248 (1960).

[14] R. D. Hazeltine and J. D. Lowrey, Phys. Plasmas 13, 14505 (2006).

[15] A. V. Filippas, R. D. Bengtson, G. X. Li, M. A. Meier, C. P. Ritz, and E. J. Powers, Phys. Plasmas 2, 839 (1995).

[16] J. C. Perez, W. Horton, K. W. Gentle, W. L. Rowan, K. Lee, and R. B. Dahlburg, Phys. Plasmas 13, 32101 (2006).

[17] D. J. Tritton, Physical Fluid Dynamics (Clarendon Press, Oxford, 1988).

[18] C. N. Baroud, B. B. Plapp, Z.-S. She, and H. L. Swinney, Phys. Rev. Lett. 88, 114501 (2002).

[19] A. Diallo and F. Skiff, Phys. Plasmas 12, 110701 (2005).

[20] A. Fujisawa, A. Ouroua, J. W. Heard, T. P. Crowley, P. M. Schoch, K. A. Connor, R. L. Hickok, and A. J. Wootton, Nucl. Fusion 36, 375 (1996).

[21] Y. Q. Zhang, A. W. DeSilva, and A. N. Mostovych, Phys. Rev. Lett. 62, 1016 (1989).

[22] F. F. Chen, Phys. Rev. Lett. 15, 381 (1965).

[23] J. Pomplun, A. G. Balanov, and E. Scholl, Phys. Rev. E 75, 040101(R) (2007).

[24] C. P. Ritz, R. D. Bengtson, S. J. Levinson, and E. J. Powers, Phys. Fluids 27, 2956 (1984).

[25] B. Li and R. D. Hazeltine, Phys. Rev. E 73, 065402(R) (2006).

[26] M. A. Meier, R. D. Bengtson, G. A. Hallock, and A. J. Wootton, Phys. Rev. Lett. 87, 085003 (2001). 


\section{Vita}

Bo Li, also named Haoqing Li, was born in Shenyang, China on November 4, 1979, the son of Jizhong Li and Guifang Hou. He graduated from Experimental High

School of Beijing Normal University in 1998 and attended Xidian University in Xi'an, China from 1998 to 2002. In July 2002, he received the degree of Bachelor of Science in physics from Xidian Unviersity. In September 2002, he entered the Graduate School of the University of Texas at Austin.

Permanent Address: No. 20 Fu Xing Lu, Beijing 100840, China

This dissertation was typeset with $\mathrm{IAT}_{\mathrm{E} X} 2 \varepsilon^{1}$ by the author.

\footnotetext{
${ }^{1} \mathrm{LTT}_{\mathrm{EX}} 2 \varepsilon$ is an extension of $\mathrm{LT}_{\mathrm{E}} \mathrm{X}$. $\mathrm{L}_{\mathrm{E}} \mathrm{T}$ is a collection of macros for $\mathrm{T}_{\mathrm{E}} \mathrm{X}$. $\mathrm{T}_{\mathrm{E}} \mathrm{X}$ is a trademark of the American Mathematical Society. The macros used in formatting this dissertation were written by Dinesh Das, Department of Computer Sciences, The University of Texas at Austin, and extended by Bert Kay, James A. Bednar, and Ayman El-Khashab.
} 